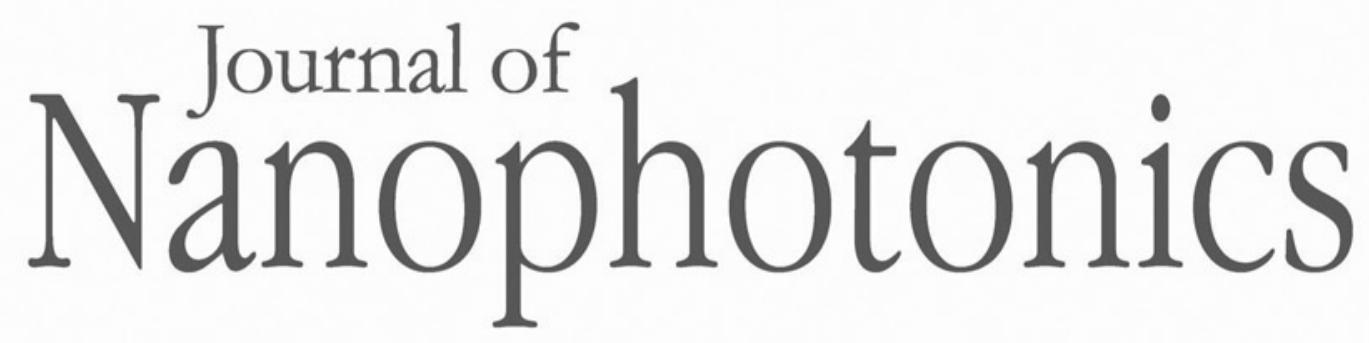

\title{
Unleashing the potential of molecular beam epitaxy grown AlGaN-based ultraviolet-spectrum nanowires devices
}

Jung-Wook Min

Davide Priante

Malleswararao Tangi

Guangyu Liu

Chun Hong Kang

Aditya Prabaswara

Chao Zhao

Latifah Al-Maghrabi

Yazeed Alaskar

Abdulrahman M. Albadri

Ahmed Y. Alyamani

Tien Khee Ng

Boon S. Ooi

Jung-Wook Min, Davide Priante, Malleswararao Tangi, Guangyu Liu, Chun Hong Kang,

Aditya Prabaswara, Chao Zhao, Latifah Al-Maghrabi, Yazeed Alaskar, Abdulrahman M. Albadri, Ahmed Y. Alyamani, Tien Khee Ng, Boon S. Ooi, "Unleashing the potential of molecular beam epitaxy grown 


\title{
Unleashing the potential of molecular beam epitaxy grown AlGaN-based ultraviolet-spectrum nanowires devices
}

\author{
Jung-Wook Min, ${ }^{\text {a Davide Priante, }}{ }^{\text {a Malleswararao Tangi, }},{ }^{\text {Guangyu Liu, }},{ }^{\text {a }}$ \\ Chun Hong Kang, ${ }^{\text {a Aditya Prabaswara, }}{ }^{\text {a Chao Zhao, }}{ }^{\text {a }}$ \\ Latifah Al-Maghrabi, ${ }^{a}$ Yazeed Alaskar, ${ }^{b}$ Abdulrahman M. Albadri, \\ Ahmed Y. Alyamani, ${ }^{b}$ Tien Khee $\mathrm{Ng}^{\mathrm{a}},{ }^{*}$ and Boon S. Ooi ${ }^{\mathrm{a}, *}$ \\ ${ }^{a}$ King Abdullah University of Science and Technology (KAUST), Photonics Laboratory, \\ Thuwal, Saudi Arabia \\ ${ }^{b}$ King Abdulaziz City for Science and Technology (KACST), National Center for \\ Nanotechnology, Riyadh, Saudi Arabia
}

\begin{abstract}
There have been recent research advances in AlGaN-based self-assembled nanowires (NWs) as building blocks for ultraviolet (UV) optoelectronics grown by plasma-assisted molecular beam epitaxy. We review the basic growth kinetics on various foundry-compatiblemetal/silicon-based substrates and the epistructure design for UV devices. We highlight the use of diffusion-barrier-metal thin film on silicon substrate as a solution to enhance device performance. NWs offer the opportunity to mitigate the detrimental quantum-confined Stark effect (QCSE), which lowers the recombination rate thereby reducing the device efficiency. On the other hand, the polarization-induced doping from the graded composition along NWs can be advantageous for eluding the inefficient doping in AlGaN-based UV devices. Sidewall surface states and the associate passivation treatment, as well as the use of ultrafast electron-microscopy characterization, are crucial investigations in shedding light on device performance under the influence of surface dangling bonds. For investigating the electrical performance of individual NWs and NWs light-emitting diode as a single entity, recent reports based on conductive atomic force microscopy measurements provide fast-prototyping in-process pass-fail evaluation and a means of improving growth for high-performance devices. Stress tests of NWs devices, crucial for reliable operation, are also discussed. Beyond applications in LEDs, an AlGaN-based NWs solar-blind photodetector demonstrated leveraging on the dislocation-free active region, reduced QCSE, enhanced light absorption, and tunable-composition features. The review opens pathways and offers insights for practical realization of AlGaN-based axial NWs devices on scalable and low-cost silicon substrates. (C) The Authors. Published by SPIE under a Creative Commons Attribution 3.0 Unported License. Distribution or reproduction of this work in whole or in part requires full attribution of the original publication, including its DOI. [DOI: 10.1117/1.JNP.12.043511]
\end{abstract}

Keywords: AlGaN; plasma-assisted molecular beam epitaxy; metal preorienting layer; metal bilayer; nanowires; epitaxy; electron microscopy; conductive atomic force microscopy; surface states; surface passivation.

Paper 18072SSV received Apr. 15, 2018; accepted for publication Jun. 21, 2018; published online Jul. 12, 2018.

\section{Introduction}

Group-III nitride compound semiconductors are regarded as the third-generation semiconductors for electronic and optoelectronic device applications. ${ }^{1-3}$ The nitride alloys and their heterostructures offer the widest tunable bandgap, spanning from the ultraviolet (UV) $(\approx 6.2 \mathrm{eV})$ to nearinfrared $(\approx 0.7 \mathrm{eV})$ spectrum. ${ }^{4-6}$ In particular, their ternary $\mathrm{AlGaN}$ and quaternary $\mathrm{Al}(\mathrm{In}) \mathrm{GaN}$

*Address all correspondance to: Tien Khee Ng, E-mail: tienkhee.ng@kaust.edu.sa; Boon S. Ooi, E-mail: boon.ooi@kaust.edu.sa 
alloys are attractive candidates for UV device applications ranging between $6.2 \mathrm{eV}$ (AlN) and $3.4 \mathrm{eV}(\mathrm{GaN})$. There are a number of immediate applications, such as UV curing, medical diagnosis and sterilization, water/air purification, gas sensing, and surface inspections within this wavelength range. ${ }^{7-10}$ Owing to the compactness and low-power consumption, AlGaN-based solid-state lighting systems are widely used in the UV-A (315 to $400 \mathrm{~nm}$ ) and UV-B ranges ( 280 to $315 \mathrm{~nm}$ ). A remarkable advantage of AlGaN UV devices is that they can replace conventional toxic and bulky mercury lamps covering the UV-C range (200 to $280 \mathrm{~nm}$ ).

AlGaN-based UV devices exhibited a less than satisfactory performance and lifetime compared with InGaN visible devices due to the poor electrical injection, which originates from difficulty in preparing p-type AlGaN material and high threading dislocation density (TDD) ${ }^{11,12}$ To mitigate the high-activation energy issue in using $\mathrm{Mg}$ as a p-type dopant in AlGaN, efforts were made in accomplishing the polarization-induced doping using composition grading in AlGaN alloys. ${ }^{13-15}$ On the other hand, the high TDD stems from the lack of economical and commercially available native substrates, which limits their use for the planar epitaxial platform. Foreign substrates, such as sapphire, $\mathrm{Si}$, and $\mathrm{SiC}$, typically result in screw-type and edge-type TDDs as high as $10^{8}$ to $10^{10} / \mathrm{cm}^{2}{ }^{16}$ To reduce TDDs by up to $\sim 10^{7} / \mathrm{cm}^{2}$, many attempts have been made to introduce low-temperature GaN or AlN buffer layers, ${ }^{17-19}$ strain-compensated buffer layers, ${ }^{20}$ pendeo-epitaxy, ${ }^{21}$ and epitaxial lateral overgrowth ${ }^{22}$ using advanced growth techniques, such as metal-organic chemical vapor deposition (MOCVD), hydride vapor phase epitaxy, and molecular beam epitaxy (MBE) ${ }^{23-26}$ Despite the current progress in the epitaxy growth, TDDs in group-III nitrides are higher than GaAs material systems having TDDs typically $<10^{2}$ to $10^{4} / \mathrm{cm}^{2}$. As a result, AlGaN-based UV light-emitting diodes (UV-LEDs) and laser diodes suffer in terms of efficiency, steady operations, and lifetime.

On the other hand, nanowires (NWs)-based epitaxial technology has distinct advantages compared with the planar platform, such as dislocation-free and reduced lattice strain, as well as lower piezoelectric polarization field and the associated smaller Stark effect in quantum-confined double heterostructures. Group-III nitride NWs, grown by plasma-assisted MBE (PA-MBE), offer the desired uniformity, density $\left(\sim>10^{10} / \mathrm{cm}^{2}\right)$, and axial growth on various heterogeneous substrates. PA-MBE growth mechanisms are solely governed by kinetics, adatom adsorption/desorption, and diffusion, which lead to self-assembled, catalyst-free NWs. In addition to PA-MBE, MOCVD is another important tool for nitride growth of both thin film and low-dimensional nanostructures. ${ }^{27}$ The growth of device quality AlGaN epilayers using MOCVD requires high growth temperatures of $1200^{\circ} \mathrm{C}$ compared with PA-MBE. This led to several disadvantages such as oxygen and aluminum contaminants, memory effect of the chamber, and limited choices of substrates for epitaxial growth. On the other hand, PA-MBE growth of $\mathrm{AlGaN}$ requires much lower temperatures (below $600^{\circ} \mathrm{C}$ to $900^{\circ} \mathrm{C}$ ), beneficial for p-type doping, and enables the use of various UV-transparent substrates, such as quartz and fused silica. In addition, due to different growth kinetics, NWs growth is self-assembled in a PA-MBE chamber without the need for an external catalyst or prepatterned substrate surface, while masks and selective area epitaxy are typically used in MOCVD growth of AlGaN NWs.

In this review, we focus on the PA-MBE grown AlGaN-based NWs platform for realizing UV devices on silicon or metal template/silicon as substrates, covering basic growth kinetics, polarization-induced doping, surface treatment, nanodevice characterization, and UV photodetection. For completeness, Table 1 lists the recent work on electrically injected AlGaN-based UV NWs light-emitting diode (NWs-LEDs) along with the figures of merit.

In Secs. 2 and 3, we present the basic growth principles of diffusion-induced self-assembled group-III nitride NWs using the PA-MBE technique. The surface morphology of a substrate is of utmost importance for ensuring a homogenous, reliable, and reproducible growth. Silicon has been widely adapted due to low-cost, abundance in supply, scalability, good thermal conductivity, and excellent crystal quality. Moreover, it is applicable to industry-related processes, thereby potentially favoring industry uptake. However, the growth of group-III nitride NWs on silicon substrates suffers from the spontaneous formation of the insulating ultrathin $\mathrm{Si}_{x} \mathrm{~N}_{y}$ layer during nitrogen plasma exposure in MBE. This is detrimental to device operation in reducing the level of current injection and heat dissipation. In Sec. 3, an overview of the recent GaN-based growth advances on metal/silicon templates, along with the insights into solving the fundamental challenges that have been faced by the group-III nitride NWs/silicon 
Min et al.: Unleashing the potential of molecular beam...

Table 1 Recent achievement in AIGaN-based UV NWs-LEDs.

\begin{tabular}{|c|c|c|c|c|c|c|}
\hline Institution (year) & $\begin{array}{l}\text { Axial top structure } \\
\text { (features) }\end{array}$ & $\begin{array}{l}\text { Power/power } \\
\text { density }\end{array}$ & EQE & $\mathrm{EL} \lambda(\mathrm{nm})$ & Substrate & $\begin{array}{l}\text { Turn-on } \\
\text { voltage } \\
\text { (V) }\end{array}$ \\
\hline Sophia Univ. $(2008)^{28}$ & Coalesced p-GaN & & & 354 & $n-S i$ & 4 \\
\hline Ohio Univ. $(2012)^{29}$ & Graded p-AIGaN & $25 \mu \mathrm{W}$ & $5 \mathrm{~m} \%$ & 336 & $\mathrm{n}-\mathrm{Si}$ & 4.5 \\
\hline McGill Univ. $(2013)^{30}$ & Axial $p-G a N$ & & & 340 & $\mathrm{n}-\mathrm{Si}$ & 5.7 \\
\hline Ohio Univ. (2014) & Graded n-AIGaN & $\begin{array}{c}0.09 \mu \mathrm{W} \\
(400 \mathrm{~mA} \text { DC) } \\
0.22 \mu \mathrm{W} \\
\text { (400 mA pulsed) }\end{array}$ & $2 \mu \%$ & 250 to 290 & $\mathrm{p}-\mathrm{Si}$ & $\sim 6$ \\
\hline Ohio Univ. $(2015)^{32}$ & $\begin{array}{c}\text { Graded n-AIGaN } \\
\text { (tunnel junction) }\end{array}$ & $\begin{array}{l}100 \mu \mathrm{W} \\
(800 \mathrm{~mA})\end{array}$ & $\begin{array}{l}4 \text { to } \\
6 \mathrm{~m} \%\end{array}$ & 310 & $\mathrm{p}-\mathrm{Si}$ & 6 \\
\hline McGill Univ. (2015) & Axial p-AIGaN & & & 207 & $\mathrm{n}-\mathrm{Si}$ & 5.5 \\
\hline McGill Univ. (2015) ${ }^{34}$ & Axial p-AIGaN & & & 210 & $\mathrm{n}-\mathrm{Si}$ & 6 \\
\hline Ohio Univ. $(2015)^{35}$ & $\begin{array}{l}\text { Graded n-AlGaN } \\
\text { (on Mo metal layer) }\end{array}$ & & & 385 & $\mathrm{Mo} / \mathrm{Si}$ & 7.2 \\
\hline McGill Univ. (2016) ${ }^{36}$ & $\begin{array}{c}\text { Axial n-GaN } \\
\text { (tunnel junction) }\end{array}$ & $\begin{array}{c}62 \mu \mathrm{W} \\
(400 \mathrm{~mA} \mathrm{DC}) \\
370 \mu \mathrm{W} \\
\text { (800 mA pulsed) }\end{array}$ & $12 \mathrm{~m} \%$ & 242 & $\mathrm{n}-\mathrm{Si}$ & 8.5 \\
\hline McGill Univ. (2016) & Axial $p-G a N$ & & & 236 to 280 & $\mathrm{n}-\mathrm{Si}$ & $>5$ \\
\hline McGill Univ. (2016) ${ }^{38}$ & $\begin{array}{l}\text { Coalesced p-AIGaN } \\
\text { (selective area) }\end{array}$ & $15 \mathrm{~W} / \mathrm{cm}^{2}$ & & 340 & $\begin{array}{c}\mathrm{GaN} \\
\text { template }\end{array}$ & 3.3 \\
\hline Ohio Univ. $(2016)^{39}$ & $\begin{array}{l}\text { Graded n-AlGaN } \\
\text { (tunnel junction) }\end{array}$ & & & 350 & Ta foil & 5 \\
\hline KAUST $(2017)^{40}$ & Coalesced p-GaN & & & 303 & $\mathrm{n}-\mathrm{Si}$ & 9 \\
\hline KAUST $(2017)^{41}$ & $\begin{array}{c}\text { Axial p-GaN } \\
\text { (on Ti metal layer) }\end{array}$ & & & 337 & $\mathrm{Ti} / \mathrm{n}-\mathrm{Si}$ & 5.5 \\
\hline KAUST $(2017)^{42}$ & $\begin{array}{c}\text { Axial p-AIGaN } \\
\text { (on Ti metal layer) }\end{array}$ & $1.9 \mu \mathrm{W} \mathrm{DC}$ & & 325 & $\mathrm{Ti} / \mathrm{TaN} / \mathrm{Si}$ & 8 \\
\hline $\begin{array}{l}\text { McGill \& Michigan } \\
\text { Univ. }(2017)^{43}\end{array}$ & $\begin{array}{l}\text { Axial p-AIGaN } \\
\text { (selective area) }\end{array}$ & $0.93 \mathrm{~W} / \mathrm{cm}^{2}$ & & 279 & $\begin{array}{c}\mathrm{GaN} \\
\text { template }\end{array}$ & 4.4 \\
\hline $\begin{array}{l}\text { Michigan Univ. } \\
(2017)^{44}\end{array}$ & $\begin{array}{c}\text { Axial n-GaN } \\
\text { (tunnel junction) }\end{array}$ & $8 \mathrm{~mW}$ pulsed & $0.4 \%$ & 275 & $\mathrm{Si}$ & $>5$ \\
\hline $\begin{array}{l}\text { Michigan Univ. } \\
(2017)^{45}\end{array}$ & $\begin{array}{c}\text { Axial } p-G a N \\
\text { (selective area/nanowall) }\end{array}$ & $0.07 \mu \mathrm{W}$ & & 214 & $\begin{array}{c}\mathrm{GaN} \\
\text { template }\end{array}$ & 7 \\
\hline $\begin{array}{l}\text { Michigan Univ. } \\
(2017)^{46}\end{array}$ & Axial h-BN & $0.05 \mu \mathrm{W}$ & & 210 & $n-S i$ & 5.5 \\
\hline KAUST $(2017)^{47}$ & $\begin{array}{c}\text { Axial p-GaN } \\
\text { (on Ti metal layer) }\end{array}$ & $3.2 \mu \mathrm{W}$ & & 332 & $\mathrm{Ti} / \mathrm{n}-\mathrm{Si}$ & 5.0 \\
\hline KAUST $(2018)^{48}$ & Axial $p-G a N$ & $1.8 \mu \mathrm{W} / \mathrm{cm}^{2}$ & & 335 & $\mathrm{n}-\mathrm{Si}$ & 9 \\
\hline
\end{tabular}


community, is presented. To this end, the growth of high-quality NWs on bulk metal substrates lays the foundation for the realization of high-power LEDs, and the use of metal foil promises roll-to-roll fabrication for eventual cost effectiveness. In this regard, we solve previously uncharted research problems by introducing a Ti/TaN preorienting layer/diffusion barrier to improve the overall band alignment and hence the carrier injection across metal/silicon, while successfully addressing the associated interface interdiffusion. The choice of TaN as a diffusion barrier, normally used for $\mathrm{Cu}$ interconnects by the dual-Damascene technique, offers the practicality of eventual implementation in Si foundry-compatible processes. Related to consumer electronics, an effort in developing high-resolution micro-LED technology based on group-III nitride was recently reported by Aledia ${ }^{49}$ for display and headset-projection applications. To this end, the NWs community may consider single-chip integration of the NWs pixel array with multicolor emission as the ultimate light source for high-resolution projection and flexible display by individually controlling each NW or each group of NWs pixel..$^{50,51}$ With production techniques reaching maturity, it is then envisaged that the economical and largescale fabrication of reliable UV light emitters based on the NWs pixel array will be feasible for eventual industrial implementation and practical applications.

Sections 4 and 5 describe the fundamental problems such as piezoelectric and spontaneous polarization fields in group-III nitrides, which highly affect the device efficiency and performance of planar structures. Here, we present using strain-free nanostructures, such as NWs, to mitigate the quantum-confined Stark effect (QCSE). An overview on the recent findings in NWs that hold promises in overcoming the issues encountered by the planar counterpart is presented. Moreover, the polarization-induced doping in AlGaN-based materials is introduced. This is of high significance as it circumvents the inefficient doping issue in both $\mathrm{n}$ - and p-types AlGaN due to high-dopant ionization energy.

Section 6 introduces the importance of the surface treatment techniques for eliminating the sidewall surface states that act as nonradiative recombination centers, thus affecting the device efficiency. This is especially important for NWs, where the surface-to-volume ratio is prominent. Passivation treatment of the surface ensures the realization of efficient and reliable devices. This effort has yet to receive the appropriate attention. We first provide a historical account of the methods employed and then introduce a highly advanced four-dimensional scanning ultrafast electron microscopy (4-D S-UEM) method to study the NWs surface carrier dynamics directly. At the same time, we elaborate and share our insights on the efficient NWs passivation techniques using both chemicals and direct-shell growth to promote carrier confinement, to reduce surface recombination velocity and improve overall optoelectronic performance.

The subsequent section focuses on the application and characterization of group-III nitride UV NWs. We view conductive atomic force microscopy (cAFM) as a powerful electrical characterization technique to enable rapid investigation cycles. The routine implementation of this technique is expected to provide immediate statistical feedback of the nanoscale and macroscopic electrical properties of the grown samples prior to the full device fabrication round. The high potential of these structures not only encompasses the material-related advantages with respect to the planar counterpart, but it allows the device miniaturization as well as enhancement in light absorption due to the nanostructure light-trapping properties. The review then ends with the report on the first UV-spectrum AlGaN-based NWs near solar-blind photodetector grown on metal template/silicon substrates.

\section{PA-MBE Growth of Self-Assembled Nanowires}

Since the first demonstrations of GaN columnar structures on sapphire and silicon substrate by PA-MBE, researchers have attempted to use the one-dimensional (1-D) columnar structures for device applications. ${ }^{52,53}$ The self-assembled GaN NW shares common N-rich (N-stable) growth conditions, which typically occur at the higher growth temperature and lower III/V flux ratio compared with planar layer growth. ${ }^{54-56}$ To keep things in perspective, we discuss the growthregime diagram suggested by Heying et al. in Fig. 1(a). It consists of three regions defined as a function of Ga flux and substrate temperatures at a constant nitrogen flux of $15.2 \mathrm{~nm} / \mathrm{min}$. It should be noted that the underlying substrate is MOCVD-grown 1.5- $\mu \mathrm{m}$-thick GaN on a $c$-plane 
(a)

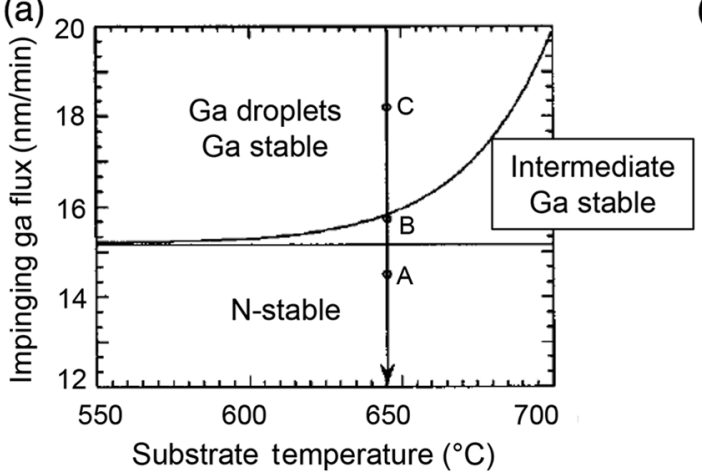

(b)

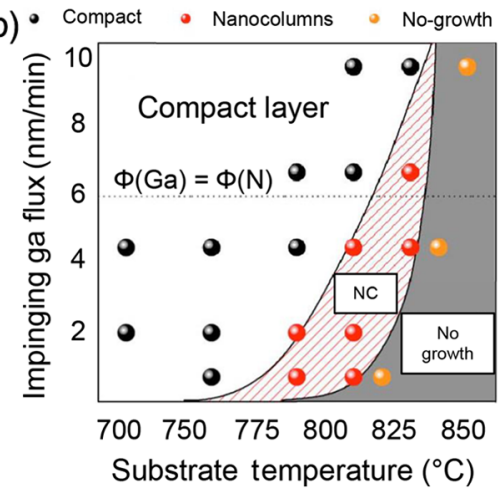

Fig. 1 (a) Surface structure diagram of $G a N$ growth depicting the $G a$ flux $\left(\Phi_{G a}\right)$ conditions plotted versus growth temperature for a constant $N$-flux $\left(\Phi_{N}\right)$ of $15.2 \mathrm{~nm} / \mathrm{min}$. Reproduced with permission from Ref. 56. (b) Growth diagram showing the growth conditions-dependent surface morphology of $\mathrm{GaN} / \mathrm{Si}(111)$ as a function of $\Phi_{\mathrm{Ga}}$ and growth temperature for constant $\Phi_{N}(6.0 \mathrm{~nm} / \mathrm{min})$. Reproduced with permission from Ref. 55.

sapphire substrate (GaN template). At low temperatures with high Ga fluxes of the "Ga-stable" regime, atomically flat and uniform $\mathrm{GaN}$ epilayers were grown on a $\mathrm{GaN}$ template in the presence of Ga droplets. With intermediate Ga fluxes at high growth temperatures, the "intermediate" regime produces $\mathrm{GaN}$ epilayers without the formation of $\mathrm{Ga}$ droplets on the surface. In an "N-stable" regime, in situ reflection high-energy electron diffraction observation revealed the characteristic spotty patterns, and this is observed for low Ga fluxes for all temperatures. Such growth conditions resulted in a columnar structure with a high density of stacking faults even when they are grown on a GaN template. In Fig. 1(b), Fernández-Garrido et al. also summarized a growth-regime diagram for GaN samples on an $\mathrm{Si}(111)$ substrate as a function of Ga flux and growth temperature at a fixed nitrogen flux of $6.0 \mathrm{~nm} / \mathrm{min}$. In this diagram, nanocolumns were grown at a high temperature with an $\mathrm{N}$-rich condition, i.e., a flux ratio of $\Phi_{\mathrm{Ga}} / \Phi_{\mathrm{N}}<1$. A $\operatorname{Si}(111)$ substrate can provide a long enough Ga adatom diffusion length for surface nucleation and three-dimensional (3-D) growth. ${ }^{55}$ The nucleation and NW growth mechanism follow the Volmer-Weber growth mode, which is induced by high lattice mismatch differences between GaN and substrate. Thus, there can be many combinations of possible substrates and buffer layers for the PA-MBE grown GaN NWs growth.

\section{Unleashing the Potential of AIGaN-Based Nanowires on Silicon, Metal, and Metal/Silicon}

In this section, we will discuss the choices of underlying substrates for AlGaN-based NWs devices and their characterizations.

\subsection{Nanowires Growth Driven by Diffusion-Induced Process}

The self-assembled NWs growth relies mainly on the diffusion-induced process. ${ }^{57}$ Taking the example of GaN NWs growth on an $\mathrm{Si}$ substrate, Ga adatoms on the surface migrate/diffuse under high substrate temperature and low III/V flux ambient. After exceeding the nucleation time, each $\mathrm{Ga}$ adatom gathers to form clusters at random locations and within the adatom diffusion length ranges. ${ }^{58}$ Then NWs start growing along axial and lateral directions, during which lateral expansion of lattice and the resultant strain relief occur. In fact, the surface energy favors adatoms diffusion along the NWs sidewalls ( $m$-planes) to selectively promote axial growth on the apex ( $c$-plane), thereby forming high aspect ratio NWs.$^{59}$ Regardless of the choice of materials in group-III nitride compounds, many researchers proved that the majority of diffusioninduced NWs have N-polar on various substrates. ${ }^{60-63}$ In spite of their high density of up to $\sim 10^{11} \mathrm{~cm}^{-2}$, NWs typically have excellent uniformity under optimum growth conditions with axially grown vertical segments for forming ternary alloys or $\mathrm{p}-\mathrm{i}-\mathrm{n}$ device structures. 
Thus, NWs devices can be fabricated in a manner similar to planar devices with meticulous process development using polymer filling and etch-back processes. ${ }^{64}$

However, ternary AlGaN NW growth properties are slightly different from GaN NW growth described above. Due to the stronger binding energy of Al-N compared with Ga-N, the diffusion length of $\mathrm{Al}$ adatoms is much shorter under the N-rich growth condition for AlGaN NWs. ${ }^{37}$ As a result, an Al-rich shell can easily form on the NWs sidewalls regardless of growth conditions. ${ }^{65}$

\subsection{Nanowires Growth on Silicon Substrate}

The earth abundant source of silicon, as well as a matured silicon substrate and process technologies, can bring about cost advantages when coupled with scaling of substrate dimension. Low resistivity, single crystalline quality of both n- and p-type Sis are ideal candidates for the growth of self-assembled AlGaN NWs and AlGaN-based NWs devices over the entire composition ranges. ${ }^{66,67}$ On top of these achievements, several research groups have demonstrated PA-MBE grown AlGaN-based NWs-LEDs emitting in the UV-A to UV-C ranges as summarized in Table 1.

However, the drawback of growing directly on silicon substrate remains. The highly reactive nitrogen plasma species typically used for PA-MBE growth result in nitridation of bare silicon at the NW/substrate interfaces as shown in Fig. 2. The formation of $\mathrm{Si}_{x} \mathrm{~N}_{y}$ can be a potential barrier for carrier injection with increasing resistance. ${ }^{68}$ Moreover, the laterally discontinuous NWsLED on Si aggravates issues with the heat dissipation problem because of a high-injection current density along the NWs. ${ }^{63}$

\subsection{Nanowires Growth on Metal Substrates}

To circumvent the above fundamental drawbacks related to growth of group-III nitride on an $\mathrm{Si}$ substrate, many researchers started growing NWs on metal. Zhao et al. ${ }^{63,70}$ demonstrated highpower InGaN/GaN LEDs on titanium (Ti)-coated bulk molybdenum (Mo) metal substrate. This scheme eliminated the formation of an $\mathrm{Si}_{x} \mathrm{~N}_{y}$ layer, while the nitrided $\mathrm{Ti}$ (TiN) conveniently functioned as a partial reflecting layer, and along with the thick molybdenum substrate, the TiN/Ti/Mo readily worked as a heat sink. The formation of TiN ohmic contact with n-GaN exhibited low turn-on voltage for InGaN/GaN NWs-LEDs. Figure 3 shows the growth evolution of GaN NWs morphology on Ti/Mo as well as the size and density of NWs achieved by varying the substrate temperature and Ga fluxes during growth. Under optimized growth condition, highdensity NWs with ordered structures were achieved. The crystalline quality and crystal structure of NWs on TiN/Ti/Mo were further confirmed in the [0002] X-ray diffraction rocking curve,

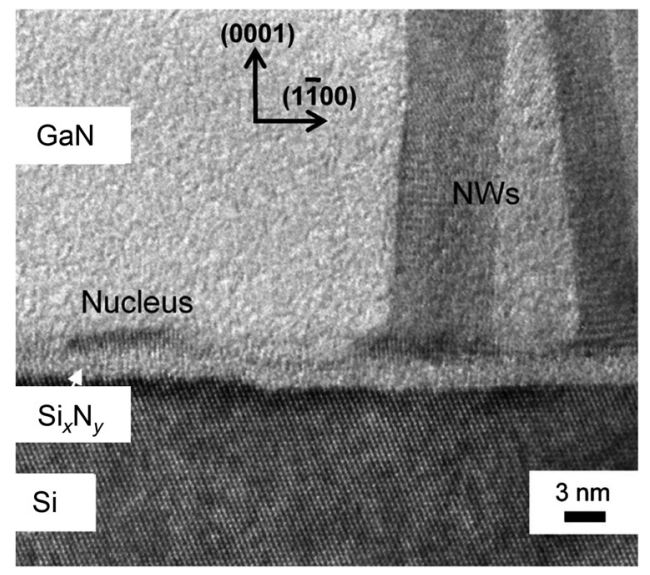

Fig. 2 Cross-sectional view of high-resolution transmission electron microscopy (TEM) image of GaN NWs on $\mathrm{Si}(111)$ showing the formation of the interfacial amorphous $\mathrm{Si}_{x} \mathrm{~N}_{y}$ interlayer. Reproduced with permission from Ref. 69. 

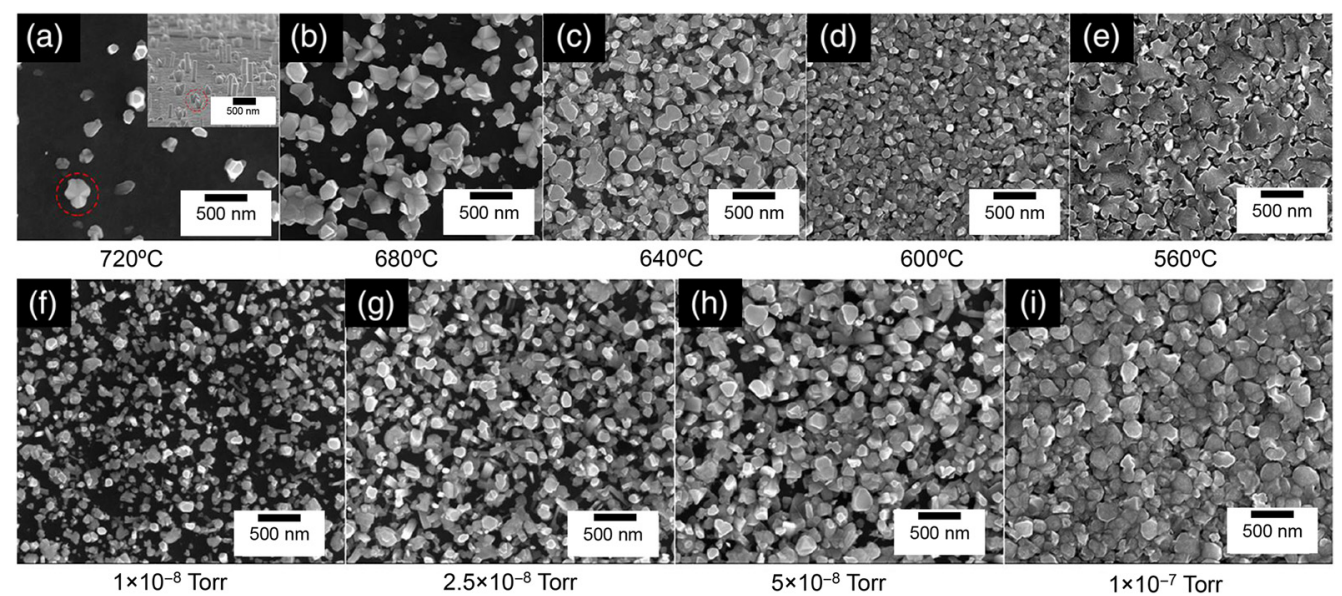

Fig. 3 (a)-(e) Plan-view SEM images for GaN NWs on Ti(500 nm)/Mo substrate with varying growth temperatures and (f)-(i) Ga fluxes at $640^{\circ} \mathrm{C}$. Reproduced with permission from Ref. 63.

while a small full-width at half-maximum value of 396 arc sec indicated that superior crystalline quality and highly ordered GaN NWs were grown on TiN/Ti/Mo. ${ }^{63}$

\subsubsection{Metal preorienting interlayer (metal thin film) on various substrates}

While the growth of group-III nitride NWs-LEDs on metal has advanced the community into higher performance devices compared with the commonly used Si substrate, bulk metals in the order of millimeters and hundreds of micrometers are considered a relatively expensive solution, despite the great prospect for the realization of high-injection and high-power conversion efficiency devices. As a preorienting layer, transition metals (Ti, Hf, Mo, Ta, etc.) can provide the preferred orientation of $c$-plane growth along the surface normal on any substrates. ${ }^{71,72}$ Moreover, the thin film deposited substrates emerge as a less expensive approach, especially for the eventual fabrication of NWs-LEDs (Table 2).

The growth of GaN NWs on these nanostructured metal layers was first reported by Rozhavskaya et al. ${ }^{73}$ A 10 - to 30-nm continuous Ti layer was deposited on a sapphire substrate, and GaN NWs were then grown using the MOCVD technique, thus achieving a high growth rate of $10 \mu \mathrm{m} / \mathrm{min}$. PA-MBE growth of GaN on a thin TiN layer on a sapphire substrate [Fig. 4(a)] was also demonstrated to prevent the chemical reaction at the materials' interface, while

Table 2 Metal preorienting layers used for group-III nitride NWs growth.

\begin{tabular}{|c|c|c|c|c|c|}
\hline Material system & Layer thickness (nm) & Device & Device structure & Central $\lambda(\mathrm{nm})$ & Ref. \\
\hline $\mathrm{Ti}$ on $\mathrm{Al}_{2} \mathrm{O}_{3}$ & 10 to 30 & - & $\mathrm{GaN}$ & 357 (UV-A) & 73 \\
\hline $\mathrm{TiN} / \mathrm{Ti}$ on $\mathrm{Si}$ & $1000(\mathrm{Ti})$ & - & $\mathrm{GaN}$ & 357 (UV-A) & 74 \\
\hline $\mathrm{TiN} / \mathrm{Ti}$ on $\mathrm{Al}_{2} \mathrm{O}_{3}$ & $1300(\mathrm{Ti})$ & - & $\mathrm{GaN}$ & - & 72 \\
\hline $\mathrm{TiN}$ on $\mathrm{Si}$ & 50 & - & $\mathrm{GaN}$ & 362 (UV-A) & 75 \\
\hline $\mathrm{ZrB}_{2}$ on $\mathrm{Si}$ & 400 & - & $\mathrm{GaN}$ & 362 (UV-A) & 75 \\
\hline $\mathrm{Ti}$ on $\mathrm{Si}$ & 50 & - & $\mathrm{GaN}$ & 363 (UV-A) & 35 \\
\hline Mo on $\mathrm{Si}$ & 50 & UV LED & GaN QW & 385 (UV-A) & 35 \\
\hline $\mathrm{Ti}$ on $\mathrm{Si}$ & 100 & UV LED & $\begin{array}{c}10 \times \mathrm{AlGaN} / \mathrm{AlGaN} \\
\mathrm{QWs} / \mathrm{QBs}\end{array}$ & 337 (UV-A) & 41 \\
\hline $\mathrm{Ti} / \mathrm{TaN}$ on $\mathrm{Si}$ & $80(\mathrm{Ti}) / 20(\mathrm{TaN})$ & UV LED & $\begin{array}{c}10 \times \mathrm{AIGaN} / \mathrm{AlGaN} \\
\mathrm{QWs} / \mathrm{QBs}\end{array}$ & 325 (UV-A) & 42 \\
\hline
\end{tabular}




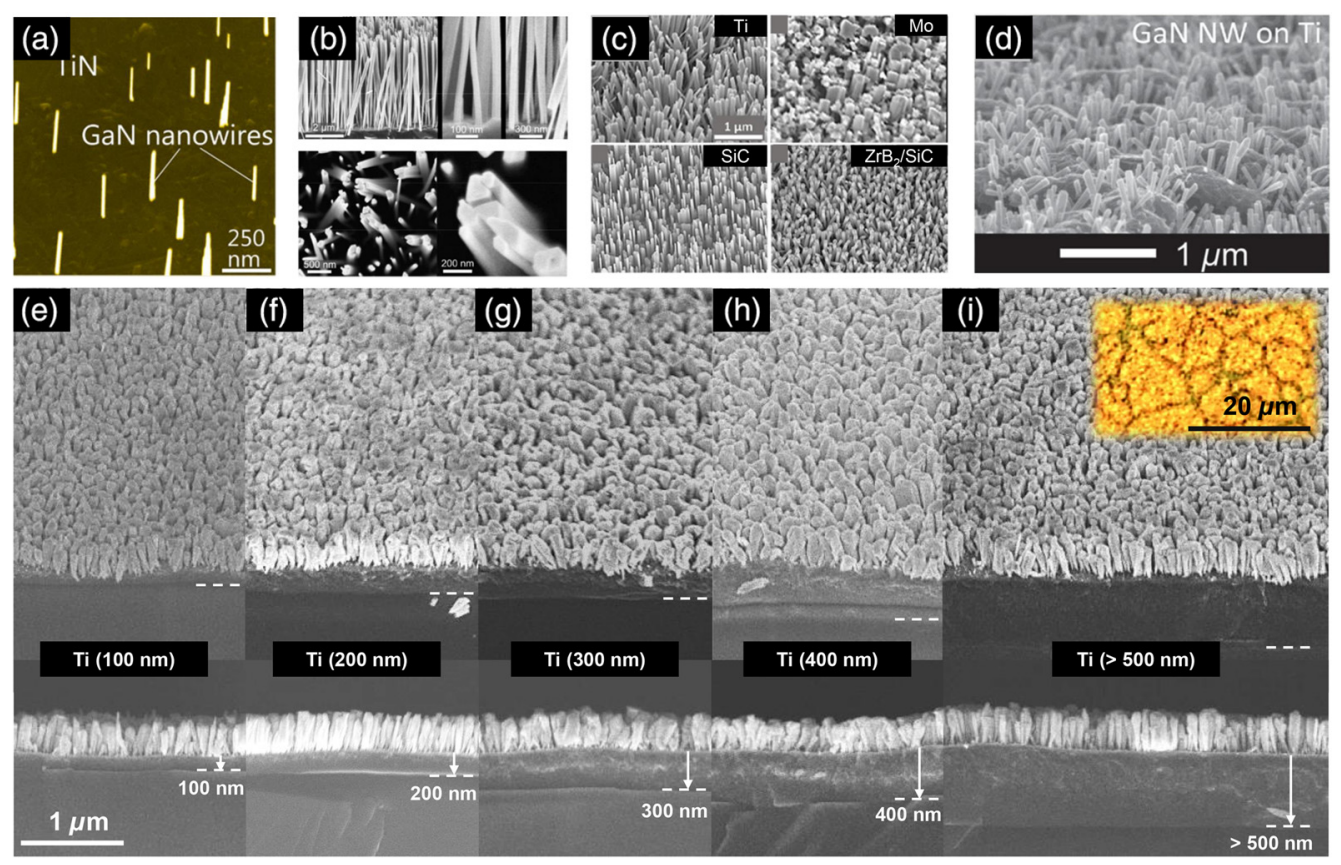

Fig. 4 GaN NWs grown on metal layer. (a) TiN thin layer. Reproduced with permission from Ref. 74. (b) SEM tilted- and top-view of GaN NWs on TiN. Reproduced with permission from Ref. 72. (c) SEM tilted-view of GaN NWs grown on Ti, Mo, SiC, and ZrB/SiC. Reproduced with permission from Ref. 75. (d) SEM tilted-view of GaN NWs grown on Ti. Reproduced with permission from Ref. 35. SEM images of tilted- and cross-sectional view of InGaN/GaN NWs of Ti thickness (e) $100 \mathrm{~nm}$, (f) $200 \mathrm{~nm}$, (g) $300 \mathrm{~nm}$, (h) $400 \mathrm{~nm}$, and (i) above $500 \mathrm{~nm}$, respectively. The inset shows the optical microscopy image of the top view of NWs on Ti with a layer thickness above $500 \mathrm{~nm}$.

simultaneously achieving high conductivity and forming ohmic contact with GaN. ${ }^{74}$ The TiN was grown due to the nitridation of the Ti layer during GaN growth using nitrogen plasma, and $\delta$-TiN was attained as confirmed using diffraction analysis. The surface, however, was irregular with trenches and bumps across the sample. Similarly, Fig. 4(b) shows the growth of $>1 \mu \mathrm{m}$ long GaN NWs on TiN thin film. Despite the uncoalesced NWs achievement, a rough surface was also obtained. ${ }^{72}$ This affects the orientation and height homogeneity, which can be detrimental to subsequent device fabrication. Serban et al. ${ }^{75}$ demonstrated a large variety of nanostructured layers, such as $\mathrm{TiN}, \mathrm{ZrB}_{2}$, Mo, and Ti thin film [Fig. 4(c)] for subsequent growth of GaN NWs, using magnetron sputtering. For devices, Sarwar et al. ${ }^{35}$ reported the first AlGaNbased UV NWs-LEDs on various nanostructured metal thin films ( $\mathrm{Ti}$ and Mo) on an Si substrate and feature of GaN NWs on Ti thin layer is shown in Fig. 4(d). Compared with a bulk Mo metal substrate, Ti and Mo thin film deposited on a 3-in. diameter Si substrate can leverage on scalability for cost reduction. Furthermore, LEDs were fabricated on Mo thin film for better electrical contact with $\mathrm{p}-\mathrm{GaN}$ compared with $\mathrm{Ti}$, while the low vapor pressure of refractory metal allowed for prolonged NW growth in an MBE growth chamber at a high substrate temperature. An electroluminescence peak at $385 \mathrm{~nm}$ was also obtained with comparable electrical performance to devices grown on an Si substrate. However, the metal thin film was found to delaminate from the Si substrates, causing rough surface and misoriented NWs. The main causes are believed to be related to the large thermal expansion coefficient mismatch between metal and silicon, as well as indirectly by oxide desorption during NWs growth. Even at lower growth temperatures for InGaN NWs, Ti layers could still delaminate. Figures 4(e)-4(i) show scanning electron microscopy (SEM) images of tilted- and cross-sectional view of n-InGaN/GaN NWs on various thicknesses of Ti layers on a 3-in. diameter Si substrate. Tilted SEM images revealed that the high density of InGaN/GaN NWs was uniformly grown on a Ti layer. An SEM crosssectional view of each interface also confirmed that, as the Ti layer thickness increased, the effects of delamination and thickness variation were aggravated. This is especially true for 
the sample with a Ti layer thickness above $500 \mathrm{~nm}$ as shown in the inset of Fig. 4(i) in which microscale cracks were observed on the surface.

This can be more severe for AlGaN NWs growth because of its higher sample growth temperatures. Compared with transition metals (Mo, $4.8 \times 10^{-6} \mathrm{~K}^{-1}$; Hf, $5.9 \times 10^{-6} \mathrm{~K}^{-1}$; Ta, $6.3 \times 10^{-6} \mathrm{~K}^{-1}$; and $\mathrm{Ti}, 8.6 \times 10^{-6} \mathrm{~K}^{-1}$ ), the thermal expansion coefficient of silicon ( $\mathrm{Si}, 2.6$ to $3.3 \times 10^{-6} \mathrm{~K}^{-1}$ ) is much lower. ${ }^{35,76}$ Eventually, a metal thin film on thick Si substrate can be partially delaminated or detached during temperature ramp up/down cycles. However, the thickness of the Ti layer was optimized to be around $20 \mathrm{~nm}$ to avoid delamination, despite that the limited conductivity at this thickness of Ti caused a higher turn-on resistance during device operation. ${ }^{77}$ The comparative study of device results as a function of Ti layer thickness allowed us to optimize the Ti thickness, which was found to be in the range of 100 to $300 \mathrm{~nm}^{78}$

(a)

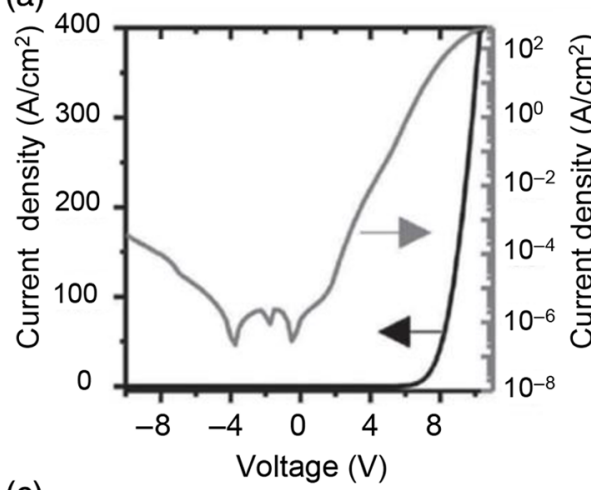

(c)

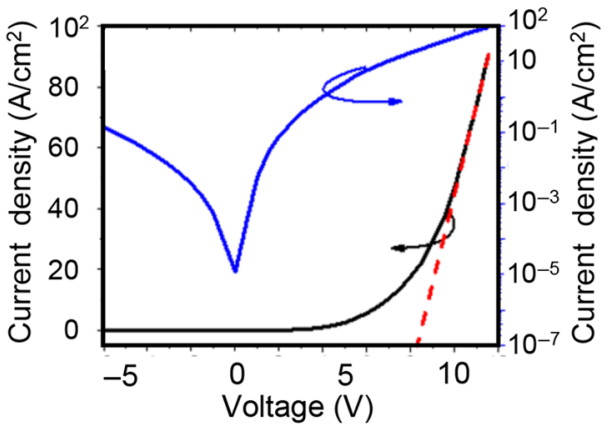

(e)

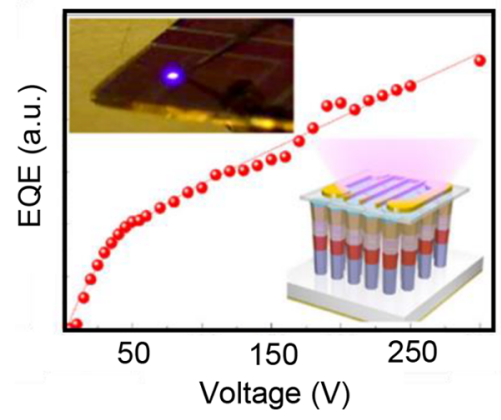

(b)

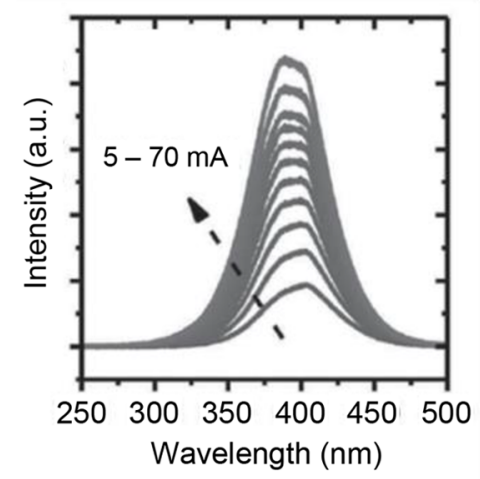

(d)

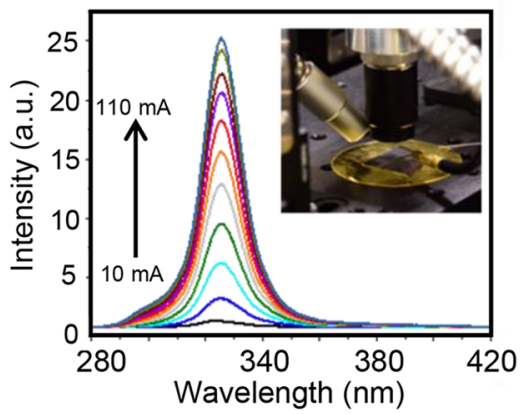

(f)

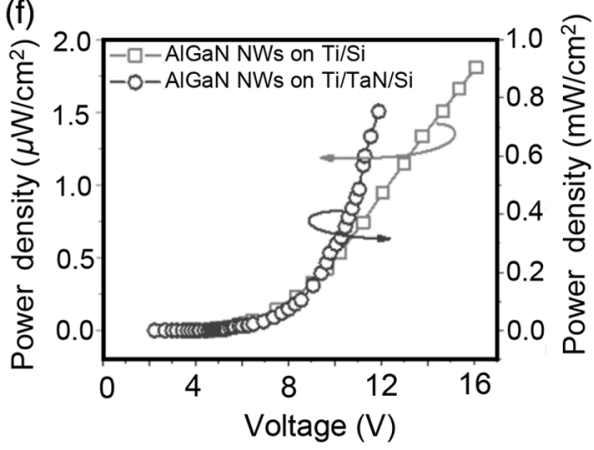

Fig. 5 UV NWs device performance. (a) Current density versus voltage characteristics of GaN NWs on Mo and (b) electroluminescence spectra at different injection currents. Reproduced with permission from Ref. 35. (c) Current density versus voltage characteristics of the AIGaN NWs-LED on Ti/TaN/Si and (d) electroluminescence spectra with the inset showing the device under test. Reproduced with permission from Ref. 42. (e) EQE versus current of the AIGaN NWs-LED on Ti/Si presenting droop-free behavior of up to $120 \mathrm{~A} / \mathrm{cm}^{2}$. Reproduced with permission from Ref. 41. (f) Power density versus voltage comparison between AIGaN-based NWs-LEDs on $\mathrm{Ti} / \mathrm{Si}$ and $\mathrm{Ti} / \mathrm{TaN} / \mathrm{Si}$. 


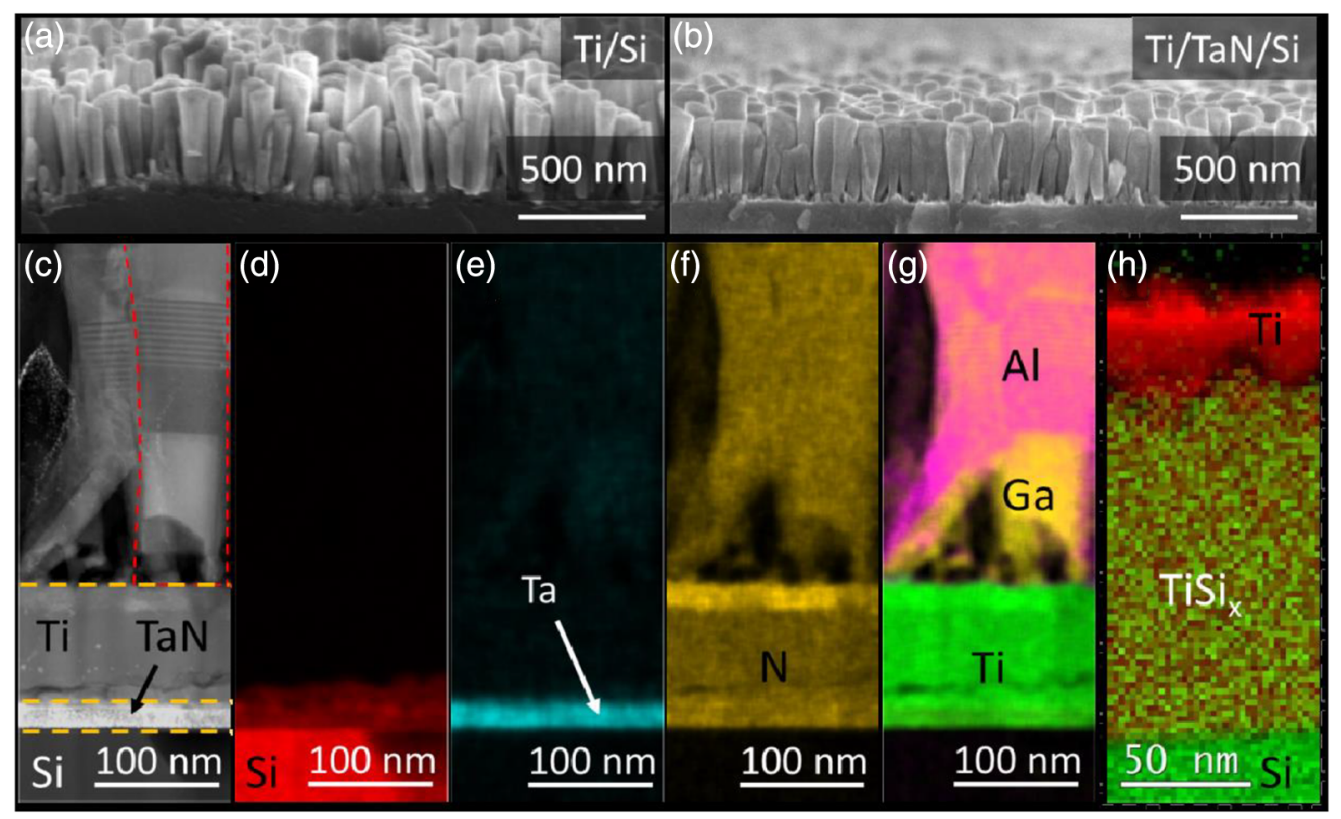

Fig. 6 (a) and (b) SEM images showing the NWs/substrate interface roughness comparison between the sample on $\mathrm{Ti} / \mathrm{Si}$ and $\mathrm{Ti} / \mathrm{TaN} / \mathrm{Si}$. (c)-(g) Dark-field cross-sectional TEM images and EDX elemental mapping of the sample on Ti/TaN/Si substrate. (h) Electron energy loss spectroscopy mapping of the sample on $\mathrm{Ti} / \mathrm{Si}$ showing the interdiffusion and $\mathrm{TiSi}_{x}$ formation, leading to the rough surface. Reproduced with permission from Ref. 42.

Along this direction, Janjua et al. ${ }^{41}$ demonstrated droop-free AlGaN-based UV NWs LEDs emitting at $337 \mathrm{~nm}$ on 100-nm-thick Ti thin film. Such a metal layer enabled the formation of TiN during nitrogen plasma exposure, hence eliminating the spontaneous formation of the $\mathrm{Si}_{x} \mathrm{~N}_{y}$ insulating layer at the NWs/substrate interface. Moreover, the reflectivity of TiN/Ti in the UV-A region (>35\%) still carries the benefit of enhanced light extraction. The external quantum efficiency (EQE) curve shows a droop-free behavior of up to $120 \mathrm{~A} / \mathrm{cm}^{2}$ due to the high carrier confinement in the quantum-disks and the role of the TiN/Ti thin film for enhanced reflection and current injection. Similar issues, however, were encountered regarding the possible partial delamination from the Si substrate and subsequent NWs misorientation. The UV NWs-LED characteristics are summarized in Fig. 5. With further TEM experimentation, we recently reported that the elevated growth temperature during MBE growth is sufficient to cause silicon to diffuse into the Ti thin film, converting $80 \%$ of the metal to $\mathrm{TiSi}_{x} .{ }^{42}$ This apparent solution was to insert a $20-\mathrm{nm}$ TaN metal interlayer between $\mathrm{Si}$ and Ti as shown in Fig. 6. The root-meansquare roughness was significantly reduced by $95 \%$ from 24 to $1.6 \mathrm{~nm}$, and improved NWs height uniformity was attained. With the TaN insertion, we demonstrated AlGaN-based NWs-LED emitting at $325 \mathrm{~nm}$ with an output power of $0.76 \mathrm{~mW} / \mathrm{cm}^{2}$ [Fig. 5(f)] and a $1000 \times$ improvement in EQE compared with the sample grown on $\mathrm{Ti} / \mathrm{Si}^{79}{ }^{79}$ The electrical conductivity was found to increase, while the sheet resistance reduced with the improved interface. Thus the integration of the metal diffusion barrier into the MBE growth process is critical to the heterogeneous growth of group-III nitride on metal/silicon. The findings, along with growth optimization and layer structure design, surface treatment and passivation, and investigations into uniform injection among all NWs, could open a research pathway toward reliable process integration and device implementation for enhanced efficiencies, low cost, and scalable widebandgap axial-NWs light emitters.

\subsubsection{Selective area growth on patterned Ti metal mask}

The NWs can be grown in a periodic manner by means of prepatterned thin $(\sim 10 \mathrm{~nm})$ titanium to form a nanohole mask. ${ }^{80}$ Therefore, the randomness of NWs morphology can be suppressed by 
(a)

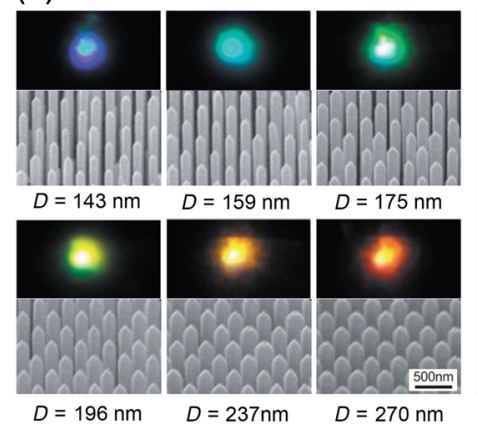

(b)

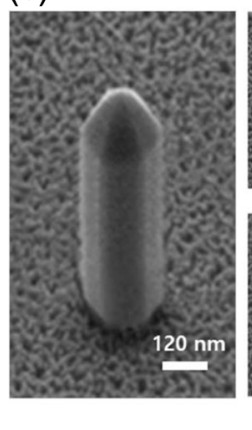

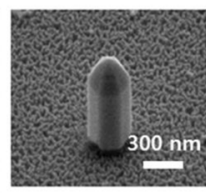
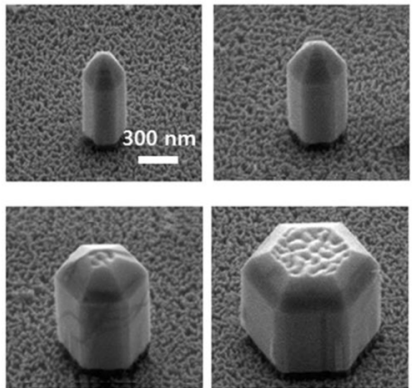

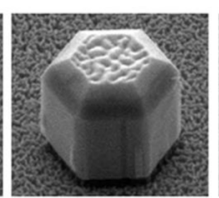

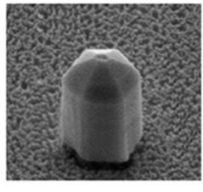

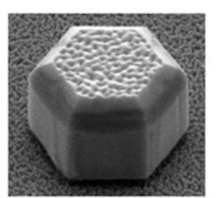

Fig. 7 (a) Tilted-view of SEM and emission images from SAG InGaN/GaN NWs as a function of different nanohole diameters. Reproduced with permission from Ref. 82. (b) Tilted-view of SEM images from InGaN/GaN single NW having various diameters. Reproduced with permission from Ref. 50.

selective area growth (SAG) of the Ti mask layer because the size and location can be controlled by titanium mask patterns as shown in Figs. 7(a) and 7(b). The key factor in SAG is the diffusioninduced process and its GaN NW growth Gemperature $\left(\sim 900^{\circ} \mathrm{C}\right) .{ }^{81}$ Sufficiently high temperature can provide longer diffusion length of $\mathrm{Ga}$ adatoms on the TiN mask, before the adatoms are trapped into the mask nanoholes. Liu et al.$^{43}$ have controlled the $\mathrm{Al}$ composition in the whole composition range to realize UV-C wavelength by SAG. Well-defined mask pattern shape, size, and spacing provided a narrower spectral line width of $\sim 11 \mathrm{~nm}$ after fabrication of AlGaN-based NW UV-C LED.

\subsubsection{Growth on metal foil}

As an alternative to the rigid substrate, recently, Calabrese et al. ${ }^{83}$ reported GaN NWs on flexible Ti foils. The NW density and morphologies were similar to the samples grown on an Si substrate. From low-temperature photoluminescence measurements, the results indicated that the $\mathrm{GaN}$ NWs on Ti foil exhibited a higher density of stacking faults due to a lower growth temperature [Fig. 8(a)]. However, a reduced density of inversion domain boundaries was also suggested. All in all, the study reveals that the flexible metal foil is also a promising candidate for optoelectronic device applications. In parallel, Myers's group succeeded in realizing AlGaN-based UV-A NWsLEDs on flexible Ta foils as shown in Fig. 8(b) ${ }^{39}$ It was suggested that large-sized roll-to-roll process could be implemented using these flexible foil substrates, which can sustain the high growth temperatures.
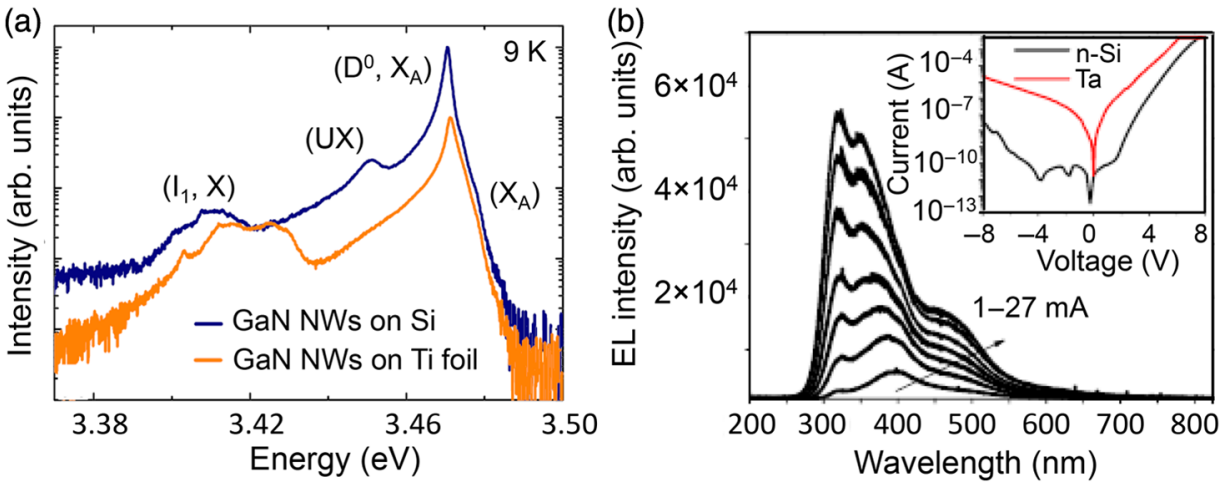

Fig. 8 (a) Low-temperature photoluminescence spectra of GaN NWs grown on Ti foil. Reproduced with permission from Ref. 83. (b) Electroluminescence spectra from AlGaN-based UV NWs-LEDs on flexible Ta foil; inset shows current-voltage characteristics of LEDs on Ta foil (red) and Si substrate (black). Reproduced with permission from Ref. 39. 


\section{4 p-Contact Layer Design for Device Structures}

Mg-doped p-GaN suffers from low hole concentration of around $1 \times 10^{-18} \mathrm{~cm}^{-3}$ due to a large dopant ionization energy of $200 \mathrm{meV} .{ }^{84}$ This can be more severe for the larger bandgap materials, such as high $\mathrm{Al}$ content $\mathrm{Al}_{x} \mathrm{Ga}_{1-x} \mathrm{~N}$ ternary alloys $\left(630 \mathrm{meV}\right.$ for $\mathrm{Mg}$-doped p-AlN). ${ }^{85}$ When using p-AlGaN as a contact layer, high resistivity can cause severe problems related to low hole injection efficiency at high-voltage operation. ${ }^{86}$ Therefore, we discuss the various top contact layer scheme with optimized thickness as well as the recent report on coalescent p-contact layer or pendeo-epitaxy contact layer.

\subsection{1 p-contact layer for axial nanowire}

Similar to planar platforms, either p-AlGaN or p-GaN axial top contacts can be used for AlGaNbased UV NWs-LEDs. The larger bandgap energy of a p-AlGaN contact offers a lower absorbance in the UV region leading to enhanced light extraction efficiency. ${ }^{33,42}$ However, due to the higher resistivity of p-AlGaN, the choice of such a p-type contact layer has its pros and cons. Janjua et al. ${ }^{48}$ optimized UV-A device structures with varying axial $\mathrm{p}-\mathrm{GaN}$ top contact thicknesses by means of simulation and experimental verification. The optimized device structure with thinner p-GaN $(20 \mathrm{~nm})$ top contact layer showed $10 \times$ improvements in output power compared with the thicker one. Mi's group also optimized the $\mathrm{p}-\mathrm{GaN}$ axial contact in conjunction with low nitrogen flow rate of around $0.4 \mathrm{sccm}$. During PA-MBE AlGaN growth, low nitrogen flow can enhance the surface migration of $\mathrm{Al}$ adatom, thus resulting in a significantly improved $\mathrm{Al} / \mathrm{Ga}$ composition. ${ }^{37}$ In this manner, a thin $(3 \mathrm{~nm})$ high-crystalline $\mathrm{p}-\mathrm{GaN}$ contact was used for AlGaN and AlN axial NWs for UV-C LEDs. ${ }^{37,45}$

Even for a very thin p-AlGaN contact, it is not completely free from the absorption of deep UV photons. ${ }^{87}$ In this regard, finding another material with a higher bandgap and better p-type conductivity is important for improving the efficiency of UV light emitters. Hexagonal boron nitride (h-BN) is one of the promising candidates for the realization of UV-C LEDs because of its ultralarge bandgap $(\sim 6 \mathrm{eV}),{ }^{88,89}$ with 10 times higher mobility ${ }^{12}$ and 10 orders of magnitude larger free hole concentration than that of AlN. ${ }^{12,90}$ Mi's group succeeded in demonstrating Mg-free AlN-based UV-C NWs-LEDs with h-BN top contact. ${ }^{46}$ Using a separate PA-MBE chamber, the h-BN top contact layer was epitaxially grown using an in situ electron-beam evaporation source under the $\mathrm{N}$-rich growth condition. With the presence of intrinsic $\mathrm{B}$ vacancies, the undoped h-BN exhibited a strong p-type conductivity, whereas the h-BN embedded AlN-based UV NWs UV-LED exhibited a strong emission at $\sim 210 \mathrm{~nm}$.

\subsubsection{Coalesced p-contact layer by pendeo-epitaxy}

The challenges in fabricating self-assembled NWs arose from the in-plane and out-of-plane tilt of crystal orientation, irregular height uniformity, and rough surface morphology. Hence, polymer-based materials have been used for planarization fabrication steps to make ohmic contact with the top semiconductor. ${ }^{40}$ However, light absorption through polymer can be severe, and the polymer does not ease heat dissipation from the already poor thermal conductivity in high $\mathrm{Al}$ content UV-LEDs. In this regard, mutually coalesced p-type contact layers atop the NWs were investigated. Kishino's group first reported the coalescence of top GaN NWs on a silicon substrate. ${ }^{91,92}$ They found that Be- or Mg-doping of GaN can remarkably enhance the lateral growth and eventually obtain a smooth and continuous layer with increasing Mg doping concentration. A similar approach was also applied to AlGaN-based UV-A NWs-LED in which the coalesced $\mathrm{p}-\mathrm{GaN}$ contact layer was successfully demonstrated with the advantage of eliminating the polymer-filling planarization step, thus simplifying fabrication. ${ }^{28}$

Janjua et al. ${ }^{40}$ also demonstrated spontaneous coalescence of $\mathrm{p}-\mathrm{GaN}$ in dislocation-free AlGaN-based UV-B NWs-LEDs on bare $\mathrm{Si}(100)$ substrate. Previously, the majority of AlGaN-based UV NWs-LED studies have been based on either polarization-induced gradedAlGaN structures or conventional double-heterostructure. However, there are limited studies on UV NWs-LEDs with an $\mathrm{Al}_{x} \mathrm{Ga}_{1-x} \mathrm{~N} / \mathrm{Al}_{y} \mathrm{Ga}_{1-y} \mathrm{~N}$ quantum-confined active region that should bring about superior carrier confinement. Having optimized PA-MBE growth for high-density 

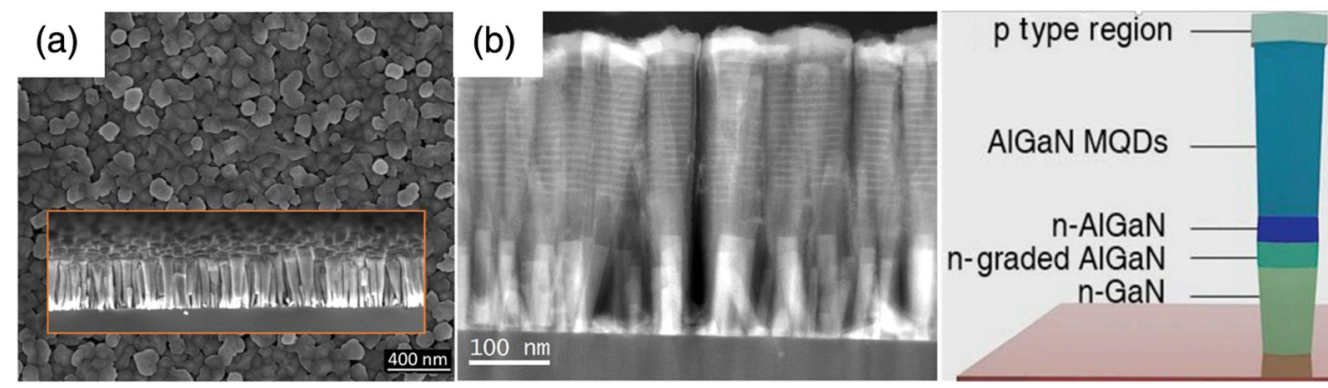

Fig. 9 (a) Top-view of SEM image and tilted-view (inset) of NWs-LED. (b) Cross-sectional high angle annual dark-field scanning transmission electron microscopy (HAADF-STEM) scan showing $\mathrm{p}-\mathrm{i}-\mathrm{n}$ AIGaN NWs with coalesced $\mathrm{p}-\mathrm{GaN}$ contact layer and 15 pairs of $\mathrm{Al}_{0.3} \mathrm{Ga}_{0.7} \mathrm{~N} / \mathrm{Al}_{0.5} \mathrm{Ga}_{0.5} \mathrm{~N}$ quantum disks/quantum barriers (left) along with a schematic representation of the layer structure (right). Reproduced with permission from Ref. 48.

NWs, spontaneously coalesced AlGaN NWs of $c$-plane orientation with a filling factor above 95\% were achieved as shown in Fig. 9(a). In Fig. 9(b), 15 pairs of $\mathrm{Al}_{x} \mathrm{Ga}_{1-x} \mathrm{~N} / \mathrm{Al}_{y} \mathrm{Ga}_{1-y} \mathrm{~N}$ quantum-confined double-heterojunction occupying half of the volume of NWs were implemented for higher probability of recombination among the injected electrons and holes in the active regions. Furthermore, the fabrication process excluded the polymer planarization step as p-contact metal can be directly deposited on the coalesced $\mathrm{p}-\mathrm{GaN}$ contact layer, thus achieving the self-planarization process. As the pendeo-epitaxy process for self-planarization was only applied to the $\mathrm{p}-\mathrm{GaN}$ layer, the $\mathrm{AlGaN}$ NWs for the remaining LED structures still possess the intrinsic properties of single-crystal NWs, such as high crystalline quality, reduced polarization field, and improved electron-hole wavefunction overlap in the active regions.

Mi's group also fabricated AlGaN-based NWs UV-A and UV-C LEDs with coalesced top AlGaN layers. ${ }^{38,43}$ Initially, mask patterns were prepared by the electron beam lithography method to produce small holes on a thin titanium layer/GaN template for growing NW arrays, thus forming the SAG template. Hexagonal openings were arranged precisely to minimize the angle of each grain boundary, while the dislocations in the coalesced top AlGaN layer were remarkably suppressed. On top of that geometry, defect-free p-AlGaN coalescent layers were grown by the SAG technique, and the Hall measurement showed free hole concentration of $7.4 \times 10^{18} \mathrm{~cm}^{-3}$ with the corresponding mobility of $8.85 \mathrm{~cm}^{2} \mathrm{~V}^{-1} \mathrm{~s}^{-1}$ at room temperature.

\subsection{Tunnel Junction and Inverted $p-i-n$ Structures}

The first tunnel diode was invented by Esaki ${ }^{93}$ in 1958 with a high-doped, extremely narrow Ge $\mathrm{p}-\mathrm{n}$ junction. Under reverse-bias, the tunnel diode showed ohmic characteristics and formed a low-resistive current path through thin n- to p-type layers. ${ }^{86}$ The property of tunnel junction makes it possible to realize multiple-stacks of various optoelectronic device structures, such as combining two active regions for dual-wavelength LED,${ }^{94}$ or high-efficiency multijunction or tandem solar cells. ${ }^{95}$ Another advantage of tunnel-junction-embedded structures is being able to convert a typical structure into $n-p-n$ LEDs in such a way that the top resistive p-type AlGaN contact is a substitute for $\mathrm{n}$-type $\mathrm{AlGaN} .{ }^{86}$ In this regards, Myers's group realized $\mathrm{n}+$ gradedAlGaN/AlGaN-quantum-disks/p+ garded-AlGaN with a bottom p-GaN/InGaN/n-GaN tunnel junction embedded on top of an n-type silicon, whereas the graded-AlGaN structures are used for inducing the respective polarization-induced doping. ${ }^{32}$ The interband tunneling at the tunnel junction allows the electron injection into the $\mathrm{n}-\mathrm{GaN}$ layer and holes into the graded p-AlGaN layer. As a result, tunnel junction LED showed reduced operating voltage and increased injection current. With $\sim 6 \mathrm{~V}$ reduction of turn-on voltage, the tunnel-junction AlGaN-based NWs-LEDs showed increased light output with wall plug efficiency comparable with a conventional AlGaN-based NWs-LED.

In additon, Mi's group integrated epitaxial $\mathrm{Al}$ metal tunnel junctions for AlGaN-based NWs ${ }^{36,44}$ as well as incorporated the spontaneously formed AlGaN shells on the sidewall for suppressing the nonradiative recombination and improving the carrier confinement. 
Compared with the conventional structures without tunnel junction, the Al tunnel junction embedded in NWs showed significant reduction in device resistance, with higher output power $(>8 \mathrm{~mW})$ and EQE $(\sim 0.4 \%){ }^{44}$

\section{Quantum-Confined Stark Effect and Strain Issues}

The group-III nitride semiconductors have been researched for light emitters over the whole UVto-near IR spectrum (AlN-InN), which subsequently led to the commercialization of the UVvisible devices. However, the significant lattice mismatch of the wurtzite structures with the different group-III nitride binary compounds grown along the polar axis leads to the formation of piezoelectric fields. This along with the spontaneous polarization fields can be of several $\mathrm{MV} / \mathrm{cm}$, thus increasing the carrier separation and QCSE, while reducing the radiative recombination (Fig. 10).

The QCSE was first reported by Miller et al. ${ }^{96}$ in the context of GaAs-AlGaAs quantum well structures with an electric field applied perpendicular to the layers. Though researchers achieved high-efficiency NWs-based LEDs, ${ }^{97,98}$ avoiding the strain and polarization-induced internal electric fields, QCSE at the interface of quantum disk/dot and barrier, remains challenging. ${ }^{99,100}$ In group-III nitride-based quantum wells, the internal piezoelectric fields of $\sim 1.2 \mathrm{MV} / \mathrm{cm}$ decrease the overlap of electron and hole wavefunctions [Figs. 10(a)] and [10(b)]. ${ }^{101,102}$ The resultant QCSE has been widely reported for III-V metal-polar quantum well-based devices ${ }^{96,101,103-}$ ${ }^{106}$ grown along the $c$-direction. This effect can be moderately mitigated in the NW geometry as a result of lateral strain relief during growth. Several efforts already have been made to understand the QCSE for GaN/InGaN-based LEDs, ${ }^{96,99,101,103-107}$ but there were limited related studies on GaN/AlN or GaN/AlGaN-based UV LEDs. ${ }^{108,109}$ In this regards, Leroux et al. reported an internal electric field strength of $0.45 \mathrm{MV} / \mathrm{cm}$ in $\mathrm{AlGaN} / \mathrm{GaN}$ structures by varying the well thickness. As the GaN well is unstrained, they attributed the origin of the field to the spontaneous polarization difference between the well and barrier, the latter being mostly affected by the piezoelectric field. ${ }^{103}$ Similarly, Lefebvre et al. ${ }^{110}$ studied the well and barrier thickness-dependent excitonic recombination dynamic and found it to be dependent on the built-in electric field.

The Al composition is known to play a crucial role in the internal electric field. Figures 11(a)-11(c) show the linear relation between the Al content and the electric field in $\mathrm{AlGaN} / \mathrm{GaN}$ quantum barrier/wells. From low-temperature photoluminescence studies, fields in the range of $1 \mathrm{MV} / \mathrm{cm}$ were reported for $25 \% \mathrm{Al} .{ }^{11,112} \mathrm{~A}$ higher electric field of 5 to $8 \mathrm{MV} / \mathrm{cm}$ was calculated by $\mathrm{Ng}$ et al. for an $\mathrm{AlGaN} / \mathrm{GaN}$ with $\mathrm{Al}$ composition of $80 \%$, with an associated large photoluminescence red shift. ${ }^{113}$ Time-resolved
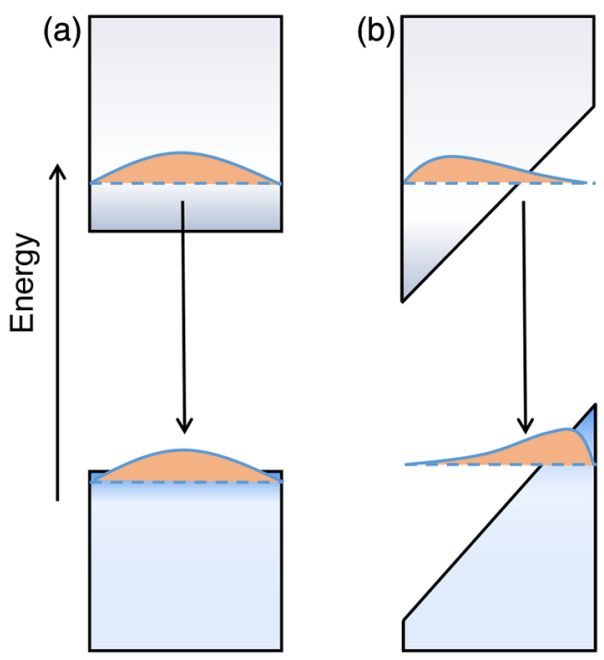

Fig. 10 The electronic band structure of the quantum well (a) without and (b) with QCSE. Effective-bandgap narrowing and spatial separation of electron and hole wavefunctions are depicted. 

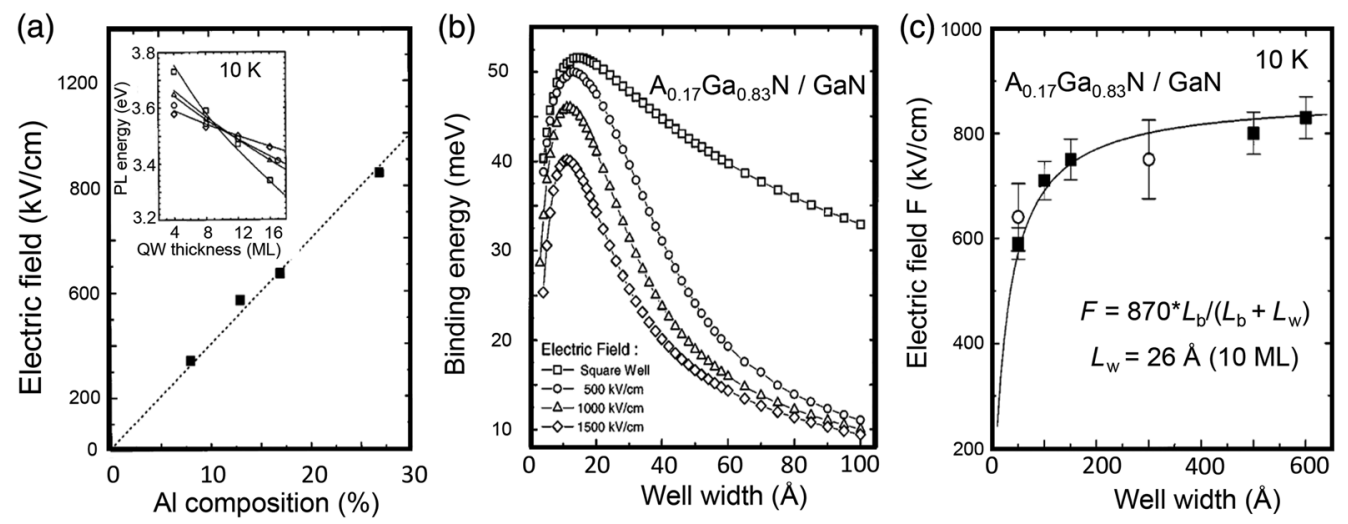

Fig. 11 (a) Built-in electric field as a function of Al composition in AIGaN/GaN quantum well structure. Reproduced with permission from Ref. 111. (b) Calculated binding energy in AIGaN/GaN structure versus GaN well thickness at different electric fields. (c) The electric field as a function of barrier thickness for the same structure as Fig. 11(b). Reproduced with permission from Ref. 112.

photoluminescence measurements were performed in two types of $\mathrm{GaN} / \mathrm{Al}_{x} \mathrm{Ga}_{1-x} \mathrm{~N}$ quantum wells. With low Al content in the barriers, an increase in the biexciton binding energy was observed with increasing quantum confinement. On the other hand, for the case of low-quantum confinement values, the biexciton binding energy value was found to be less than that of the bulk $\mathrm{GaN}$ for $\mathrm{GaN} / \mathrm{Al}_{0.05} \mathrm{Ga}_{0.95} \mathrm{~N}$ quantum well. This evidenced the presence of the strong QCSE. ${ }^{114}$ Other than simple quantum well/quantum barrier studies, the piezoelectric effects on $\mathrm{AlGaN} / \mathrm{GaN}$ laser structures were also analyzed by means of simulation. The piezoelectric effects affect the device performance (C1-HH1 and C1-LH1 gain transitions) at a relatively low carrier density $\left(<10 \times 10^{19} \mathrm{~cm}^{-3}\right)$, implying that the free carrier screening effect is not prominent. However, above this threshold, a flat-band configuration was obtained. ${ }^{115}$ To suppress the QCSE, AlGaN/GaN structures were grown on semipolar and nonpolar substrates, i.e., with a growth axis at an angle perpendicular to the (0001) substrate axis. Improved photoluminescence was achieved comparing structures grown on (0001) and $(11 \overline{2} 0)$ with a 20 to 30 times enhancement for the latter, signifying the reduction of the internal field. ${ }^{116}$ Strong red shift and reduced photoluminescence intensity were obtained for $\mathrm{AlGaN} / \mathrm{GaN}$ structures grown on $c$-plane sapphire, compared with $m$-plane GaN substrates with internal electric fields as high as $1.23 \mathrm{MV} / \mathrm{cm}$ for the former. ${ }^{117}$

To understand the role of the internal piezoelectric field induced QCSE on photoluminescence peak position of quantum disk embedded in NWs, one must determine the direction of the electric field, which is related to the strain-induced polarization. From the elastic theory of wurtzite materials, the relation between strain and the piezoelectric polarization expressed in the following is used to determine the direction of polarization ${ }^{101}$

$$
P_{c}=2 d_{31}\left(C_{11}+C_{12}-\frac{2 C_{13}^{2}}{C_{33}}\right) \varepsilon_{a},
$$

where $P_{c}$ is the polarization along $c$-direction, $d_{31}$ is the piezoelectric modulus, $C_{11}, C_{12}, C_{13}$, and $C_{33}$ are the elastic constant, and $\varepsilon_{a}$ is the in-plane compressive strain $(<0)$. Hence, for an $\mathrm{AlGaN}$ (quantum disk)/AlN (quantum barrier) system, strain-induced polarization is positive $\left(P_{c}>0\right)$ pointing along [0001]. Consequently, the direction of the piezoelectric field in the N-polar (metal-polar) devices directs along [0001] ([0001]). Thereby, for AlGaN-based UV devices, the direction of the internal electric field induced by the piezopolarization points from the substrate to the surface for N-polar, whereas it points from the surface to the substrate for metalpolar. Therefore, the QCSE has deleterious effects on the device performance by reducing the rate of recombination, whereas it has less of an impact on cubic and nonpolar wurtzite devices. ${ }^{118,119}$ In terms of nanostructures, Renard et al. ${ }^{100}$ demonstrated the existence of the QCSE for the polar GaN/AlN axial heterostructures in NWs using temperature-dependent 
photoluminescence and single-photon correlation measurements. They reported that the magnitude of QCSE was observed to be small compared with that of two-dimensional (2-D) structures, which were ascribed to the reduction in piezoelectric polarization accompanied by the elastic relaxation. Songmuang et al. ${ }^{109}$ reported the photoluminescence transition energy as a function of both the quantum dot height and the $\mathrm{Al}$ mole fraction in the barriers, as well as the evolution of the decay time as a function of the quantum dot height; thereby, they found that a built-in electric field is remarkably smaller than the value expected from the spontaneous polarization. On the other hand, Wang et al. ${ }^{120}$ observed the suppression of QCSE for nanorodbased device structures formed by a top-down approach. Later, the coexistence of QCSE along with localized states for InGaN/GaN NW heterostructure having the emission centered at around $2.5 \mathrm{eV}$ has been demonstrated. ${ }^{99}$

Müßener et al. ${ }^{121}$ reported the direct analysis of polarization-induced internal electric fields in single $\mathrm{GaN} / \mathrm{Al}_{0.3} \mathrm{Ga}_{0.7} \mathrm{~N}$ quantum-disks embedded in GaN/AlN NW heterostructures. Superposition of an external electric field with different polarities results in compensation or enhancement of the QCSE in the quantum disks. As shown in Fig. 12(a), a blue shift (red shift) in PL emission energy and an increase (decrease) in PL intensity were observed for positive (negative) bias, i.e., for compensation (enhancement) of the polarization-induced internal electric field and the resulting QCSE. The results revealed the NW heterostructures crystal polarity ( $\mathrm{N}$-face) and allowed determination of the strength of the internal electric fields of $1.5 \mathrm{MV} / \mathrm{cm}$. By field-dependent analysis of the low-temperature photoluminescence energy and intensity, we proved the $[000 \overline{1}]$-polarity of the NW and determined the internal electric field strength of $1.5 \mathrm{MV} / \mathrm{cm}$. Recently, Tangi et al. ${ }^{62}$ reported the bias-dependent photoluminescence exhibiting opposite shifts in contrast to that of the planar quantum well structures, which were attributed to the change in the direction of the piezoelectric field for N-polar NWs [Fig. 12(b)]. Furthermore, the blue shift of photoluminescence with increasing excitation power reveals the suppression of QCSE resulting from the photogenerated carriers. Thus the NWs ensemble LED device results are in good agreement with the single NW device in terms of the bias controlled photoluminescence shifts and variation of the intensity. Carnevale et al. ${ }^{29}$ observed electroluminescence peaks at various wavelengths from $250 \mathrm{~nm}$ in $\mathrm{Al}_{0.8} \mathrm{Ga}_{0.2} \mathrm{~N}$ quantum disk LEDs to $360 \mathrm{~nm}$ in $\mathrm{GaN}$ quantum disk LEDs. The conductivity and electroluminescence showed slight variation with temperature, verifying that the electrons and holes are polarization induced, rather than thermally ionized from impurities. Since polarization charge is built into the unit cell, the NW doping profile in the devices, in the absence of impurity doping, is uniform unlike impurity-doped NWs.
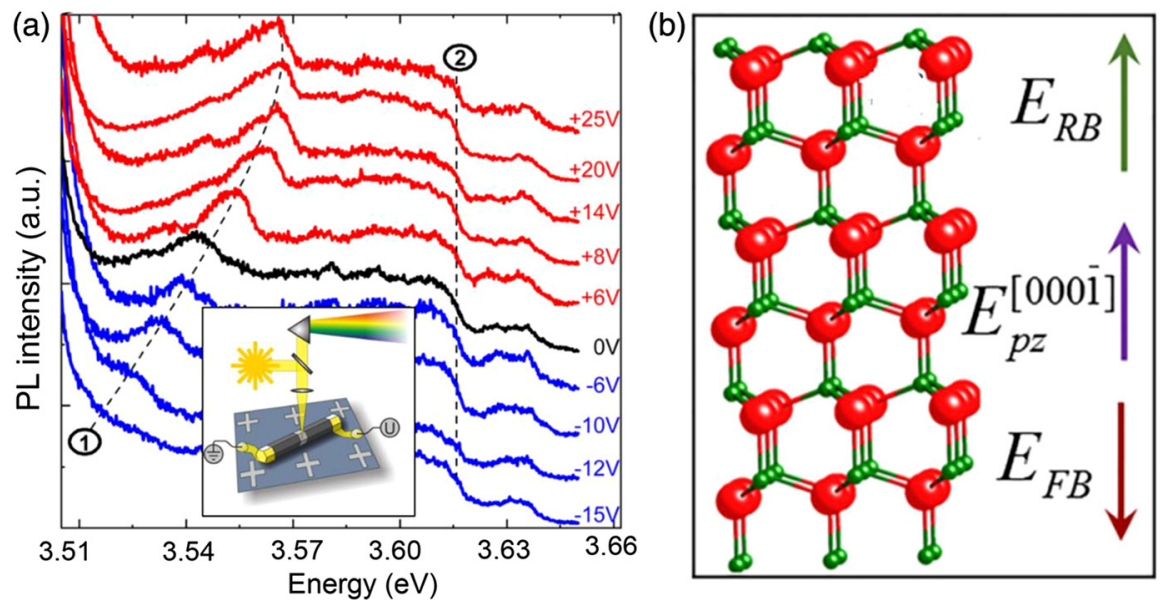

Fig. 12 (a) Photoluminescence spectra under the influence of an external bias focusing on the energy range above the GaN near band edge emission. The shifting and fixed (strained GaN related) spectral features are labeled 1 and 2, respectively. The spectra are normalized to the quantum-disks emission and vertically shifted for clarity. The inset shows the schematic representation of a single NW excited with a laser line. Reproduced with permission from Ref. 121. (b) The direction of internal piezo and external electric field with respect to the [0001] direction of $\mathrm{N}$-polar wurtzite crystal structure. Reproduced with permission from Ref. 62. 


\section{Doping in AIGaN Nanowires}

The noncentrosymmetric wurtzite crystal structure of group-III nitride compound semiconductors leads to the spontaneous and piezoelectric polarization, which accumulates the net charge at surfaces and interfaces. ${ }^{14}$ Utilizing the polarization-induced charge, researchers observed that a graded heterojunction of AlGaN establishes a 3-D slab of bound charge that can provide either an n- or p-type conducting layer depending on the sign of the Al concentration gradient and the polarity of the material. Jena et al. ${ }^{13}$ demonstrated the realization of polarization-induced bulk electron doping in III-V nitride semiconductors by grading the AlGaN/GaN heterojunction over a distance of $\approx 100 \mathrm{~nm}$. The polarization-induced bulk charge (3-D electron gas), mimicking a local donor with zero activation energy, overcomes the localized optical transitions associated with the defect centers of donor atoms. Such carriers do not freeze out at low temperature, unlike the shallow donor-doped bulk carriers, and thus exhibit high mobilities due to the absence of ionized impurity scattering. Figures 13(a) and 13(b) show the temperature-dependent mobility and carrier sheet densities for the Si-doped GaN samples, 100-nm linearly graded (0\% to 30\% Al) $\mathrm{AlGaN}$ and $20-\mathrm{nm} \mathrm{Al}_{0.20} \mathrm{Ga}_{0.80} \mathrm{~N} / \mathrm{GaN}$. The carrier in the $0 \%$ to $30 \%$ graded $\mathrm{AlGaN}$ sample mimics the transport characteristics of modulation-doped 2-D electron gas (2DEG) and 3-D electron slab (3DES) characterized by a lack of activation energy, leading to a temperature-independent carrier density. Carriers in the bulk donor-doped sample show the characteristic freeze-out associated with the hydrogenic shallow donor nature of $\mathrm{Si}$ in bulk GaN. A theoretical dopant activation fitting yielded an activation energy of $20 \mathrm{meV}$ with a doping density fixed by the Si flux in MBE $\left(10^{18} / \mathrm{cm}^{3}\right)$. The wide slabs of high-density mobile electrons were created without introducing shallow donors. Transport measurements revealed the superior properties of the polarization-doped electron distributions over the comparable shallow donor-doped structures, especially at low temperatures due to the removal of ionized impurity scattering. Thus this clearly demonstrated the improvement in the conductivity of polarization-doped layers over comparable donor-doped layers. ${ }^{13}$ Later, the polarization-induced doping method was utilized in the case of NWs for obtaining sufficient doping levels.

Later, Simon et al. ${ }^{14}$ showed the high-efficiency p-type doping by ionizing acceptor dopants (Mg) by employing the built-in electronic polarization in bulk uniaxial wide-bandgap wurtzitesemiconductors. The holes created by this method are field-ionized and are strong enough to withstand the thermal freeze-out effects, which results in superior p-type electrical conductivity. This doping scheme helped to improve the optical emission efficiency in UV-LED structures. Thus the polarization-induced doping renders a solution to both $\mathrm{p}$ - and n-type doping issues in wide-bandgap AlGaN semiconductors. The doping scheme can be used to obtain the desired hole or electron concentrations in spite of poor ionization efficiencies of impurity dopants in
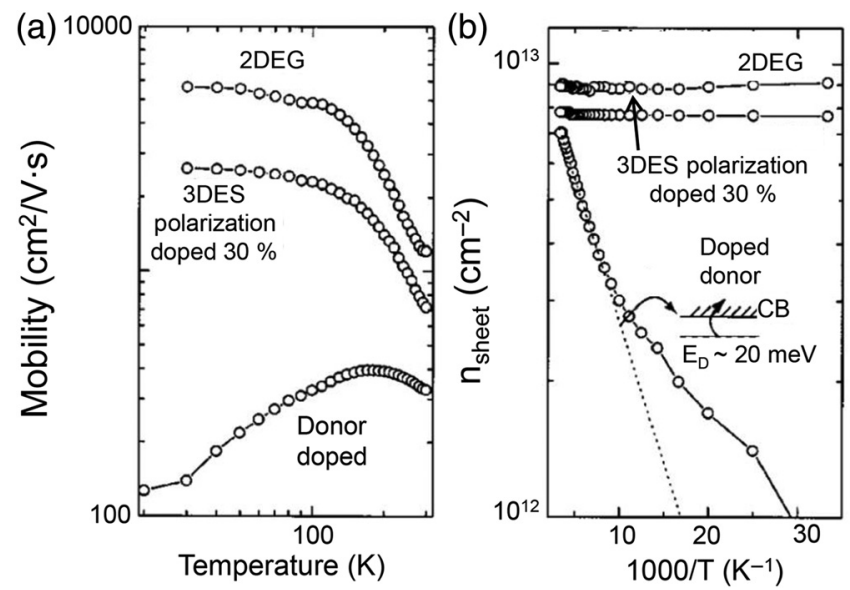

Fig. 13 The temperature-dependent (a) mobility and (b) carrier sheet densities for a polarizationdoped sample of 3DES, donor-doped sample, and a 2DEG sample structures. Reproduced with permission from Ref. 13. 

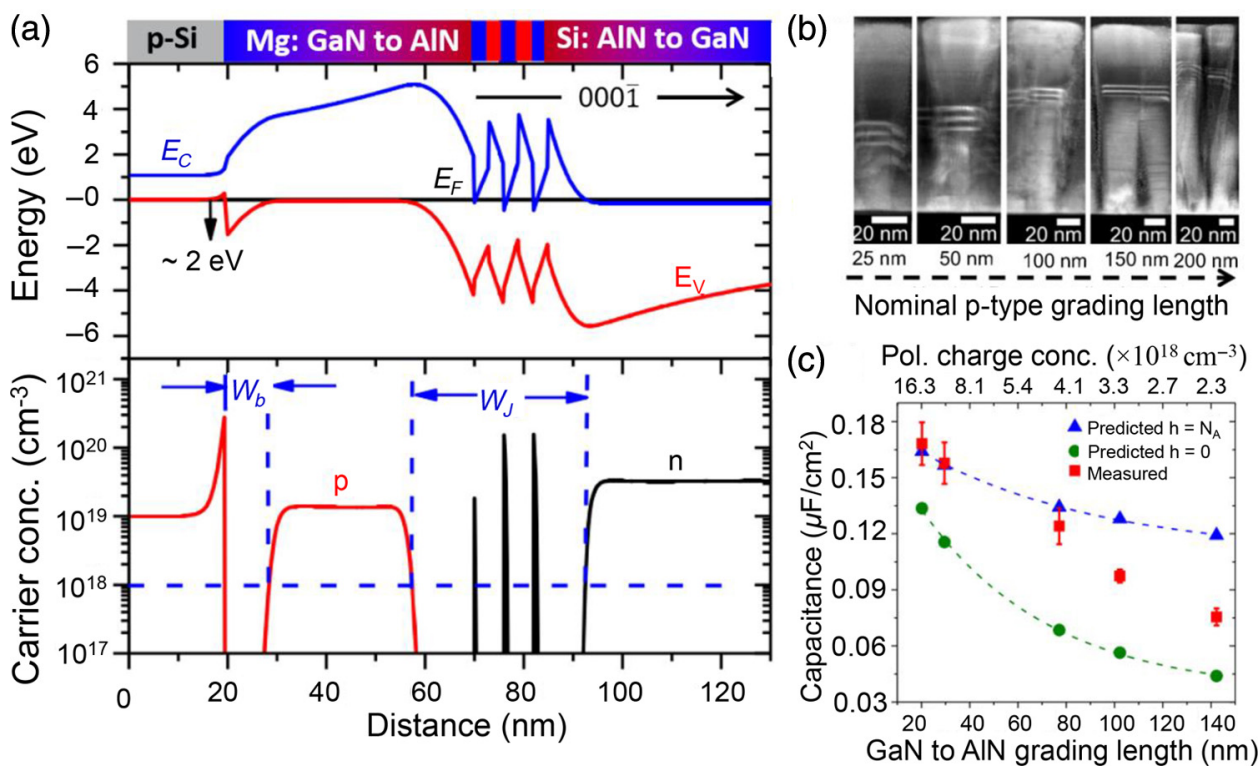

Fig. 14 (a) Schematic of NW heterostructure on (top) p-type Si and (bottom) energy band diagram and carrier concentration profile along the axis of the NW heterostructure considering spontaneous and strain-induced polarization charge. (b) STEM images of graded NW heterojunctions. (c) The calculated capacitance from a single 1-D circuit model (triangles predicted with $h=N_{A}^{\text {pol }}$ ) and assuming full depletion (circles predicted with $h=0$ ) are shown. The top horizontal axis corresponds to the negative bound spontaneous polarization charge density calculated from the measured concentration gradient. The square symbols represent the extracted 1-D NW capacitance from measured capacitance. Reproduced with permission from Ref. 15.

any semiconductor crystals that possess sufficiently strong spontaneous and piezoelectric polarization.

Recently, Myers's group reported the tuning of polarization-induced hole density in the NWs graded from GaN to AlN using capacitance-voltage (CV) measurements. Figure 14(a) shows the schematic of NW heterostructure on p-type Si (top), energy band diagram, and carrier concentration profile (bottom) along the axis of the NW heterostructure. Figure 14(b) shows the STEM images of graded NW heterojunctions. Figure 14(c) shows the calculated capacitance from a simple 1-D circuit model (triangles predicted with hole concentration, $h=N_{A}^{\mathrm{pol}}$ ) and assuming full depletion (circles predicted with $h=0$ ). The top horizontal axis corresponds to the negative bound spontaneous polarization charge density calculated from the measured concentration gradient. The squares are the extracted 1-D NW measured capacitance. Their experiments revealed that the negative polarization-induced bound charge is partially compensated for by both free holes and deep donors available near the valence band for concentration gradients of $<1.30 \% \mathrm{Al} / \mathrm{nm}$. On the other hand, for gradients $\geq 1.30 \% \mathrm{Al} / \mathrm{nm}$, the deep donor concentration is negligible and free hole concentrations roughly equal to the bound polarization charge density of up to $1.6 \times 10^{19} \mathrm{~cm}^{-3}$ for a concentration gradient of $4.95 \% \mathrm{Al} / \mathrm{nm}^{15}$

The traditional impurity-induced doping of $\mathrm{AlGaN}$ suffers from the large activation energy of $\mathrm{Mg}$ and high dislocation density of AlGaN thin film. Compared with the planar counterpart, the $\mathrm{Mg}$ incorporation in $\mathrm{AlGaN} \mathrm{NW}$ is more efficient because of the lower formation energy. ${ }^{122,123}$ Moreover, the hole hopping conduction in Mg impurity bands leads to the smaller activation energy in AlGaN NWs compared with thin film. ${ }^{45}$ Based on this, Myers's group and Mi's group demonstrated AlGaN-based UV LEDs. ${ }^{124,125}$ Moreover, Mi's group reported low-threshold UV random lasers on Si by combining the Anderson localization of light and high-quality AlGaN NWs array. ${ }^{126}$ In addition to demonstrating UV emitters, we have also quantified the hole concentration in AlGaN NWs using photoelectrochemical measurements. The optimized doping for AlGaN-based UV NWs-LEDs favors low turn-on voltage and low self-heating. ${ }^{127}$ 


\section{Surface Treatment}

One of the main challenges of GaN-based devices is the high concentration of growth-related defects that affect the optimum device performance. These defects act as nonradiative recombination centers, trapping the carriers and preventing the direct recombination process from happening, thus hampering the quantum efficiency, threshold current, radiative lifetime, and reliability. It is then of fundamental importance to understand the deep level incorporation for the growth of high-quality materials. Deep level transient spectroscopy (DLTS) and deep level optical spectroscopy (DLOS) are the essential techniques for analyzing the deep level defects within group-III nitride semiconductors. AlGaN/GaN heterostructures were studied, and bandgap states at $E_{c}-3.85 \mathrm{eV}$ for $\mathrm{AlGaN}$ and $E_{c}-2.64 \mathrm{eV}$ and $E_{c}-3.30 \mathrm{eV}$ for GaN were observed. ${ }^{128}$ Moreover, the defects in ammonia-based and N-plasma-based MBE growth of $\mathrm{GaN}$ have been studied. An increase in the concentration for the energy levels at $E_{c}-3.28 \mathrm{eV}, E_{c}-2.6 \mathrm{eV}$, and $E_{c}-0.60 \mathrm{eV}$ was observed, while similar main defect species of $\mathrm{C}$ and $\mathrm{Ga}$ vacancies were observed in both techniques of MBE growth. ${ }^{129} \mathrm{Mg}$-doped GaN planar layers were also analyzed by DLOS. Deep levels at $E_{v}+3.05 \mathrm{eV}, E_{v}+3.22 \mathrm{eV}$, and $E_{v}+3.26 \mathrm{eV}$ were attributed to deep donor-acceptor pair luminescence band and $C_{\mathrm{Ga}}$, respectively. ${ }^{130} m$-plane and $c$-plane GaN were also compared, revealing significant differences in both energy level or defect states and defect concentration. $10 \times$ to $50 \times$ higher defect concentration was measured using both DLTS and DLOS for the $m$-plane GaN sample. ${ }^{131}$

GaN NW surface is dominated by the effects of Ga dangling bonds and $\mathrm{N}$ vacancies, and treatment of this defective surface has been thoroughly studied in optoelectronic devices. $\mathrm{SiO}_{x}$ and $\mathrm{Si}_{x} \mathrm{~N}_{y}$ are frequently used as contact isolation layers to reduce leakage current, avoiding the contact with air and reduce GaN surface band bending. ${ }^{132,133}$ Among other uses, $\mathrm{SiO}_{x}$ has also been used to selectively block the threading dislocation in epitaxially grown LED structures to increase the quantum efficiency ${ }^{134}$ and contribute to increasing the output power of LED with an optimized refractive index gradient. ${ }^{135} \mathrm{Al}_{2} \mathrm{O}_{3}$ is another passivation material for improving the blue LED brightness ${ }^{136}$ and reducing the leakage current. ${ }^{137}$ Double layer $\mathrm{Al}_{2} \mathrm{O}_{3} / \mathrm{SiO}_{x}$ demonstrated the higher power output by simultaneously passivating the GaN surface and increasing the critical angle of the light escape cone. ${ }^{138}$

For III-V semiconductors, other than thin dielectric film protection, the surface state treatment on thin film GaAs mostly involved sulfur-based compounds, such as ammonium sulfide and sodium sulfide. ${ }^{139}$ Moreover, prior Ga-O removal allows the effective passivation of dangling bonds using sulfur-based compounds by creating $\sigma$ bond between $\mathrm{S}$ and $\mathrm{Ga}$, thus reducing the net charge accumulation on the surface. ${ }^{140}$ Sulfur passivation covalently satisfies all Ga and As dangling bonds to shift the energy bands of the surface states toward the conduction band to reduce charge traps.

The sidewalls of group-III nitride NWs possess a large specific surface with a high density of surface states due to Ga dangling bonds, nitrogen vacancies, and/or surface oxides. ${ }^{143,144}$ This results in surface Fermi level pinning and the subsequent energy band bending on the NW surface, which increases the Shockley-Read-Hall (SRH) nonradiative recombination. The excited carriers may pass through an intermediate energy state induced by the localized defects, ${ }^{64}$ and other nonradiative recombination sites, thereby resulting in a reduction in the light-emitter performances. ${ }^{107}$ To solve this problem, we have recently demonstrated that the use of long-carbon chained octadecanethiol (ODT) surface treatment of group-III nitride NWs-LED improves the light emission intensity due to a reduction in the SRH recombination and an increase in carrier lifetime. ${ }^{64}$ InGaN/GaN NWs were soaked in a mix of ODT, ethanol, and $\mathrm{NH}_{4} \mathrm{OH}$ and kept at $60^{\circ} \mathrm{C}$. Raman spectroscopy measurement showed the blue shift of the SO mode for the ODT-passivated sample, compared with the unpassivated one, confirming the alteration of the surface dynamic charge and the effectiveness of the surface treatment. As a result, there was a $\sim 4$ enhancement in PL intensity as well as a $\sim 1.5 \times$ increase in EQE. Figures $15(a)$ and 15(b) show the photoluminescence and EQE comparison of the passivated and unpassivated samples.

Although photoluminescence and EQE enhancement provide qualitative information on the passivation effects, the direct visualization of the surface carrier dynamics is necessary and can be achieved by employing a 4-D S-UEM technique. The time-resolved technique is the only 

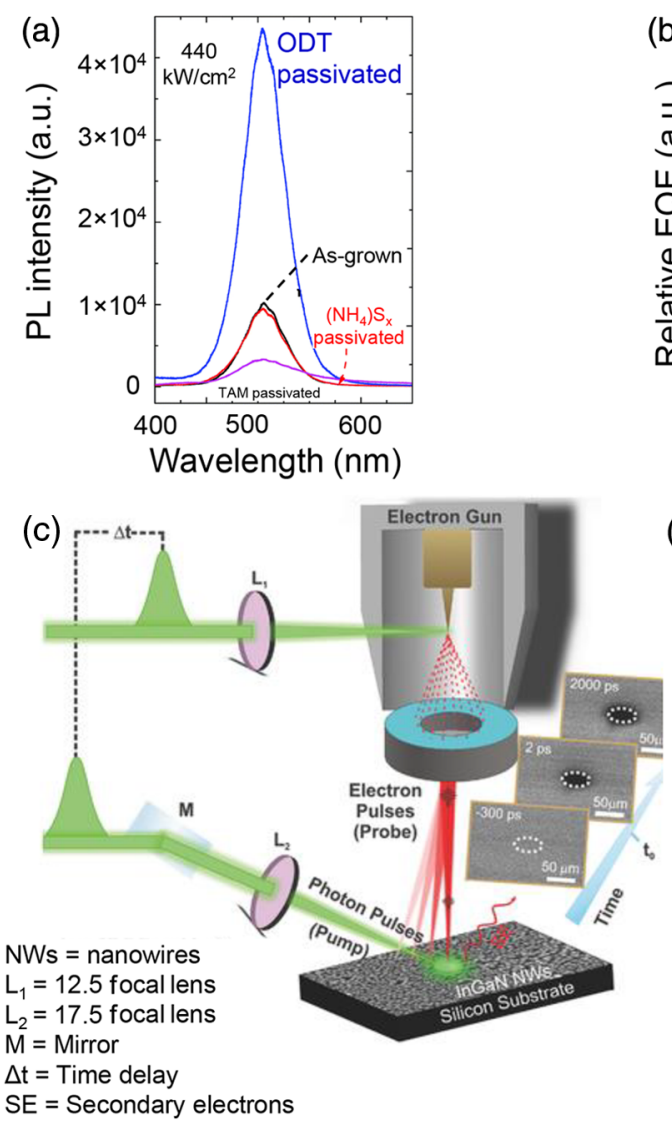

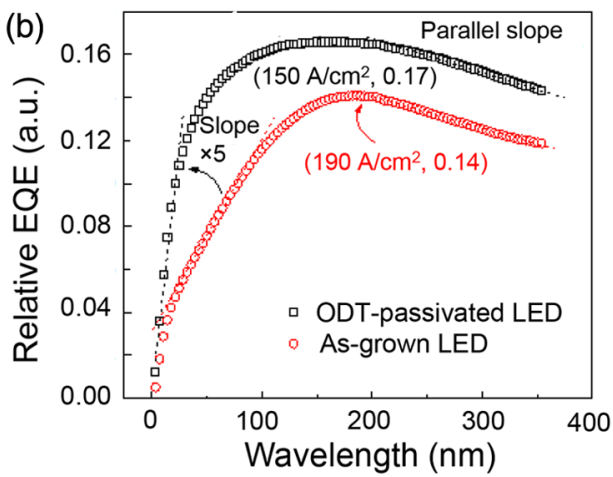

(d)

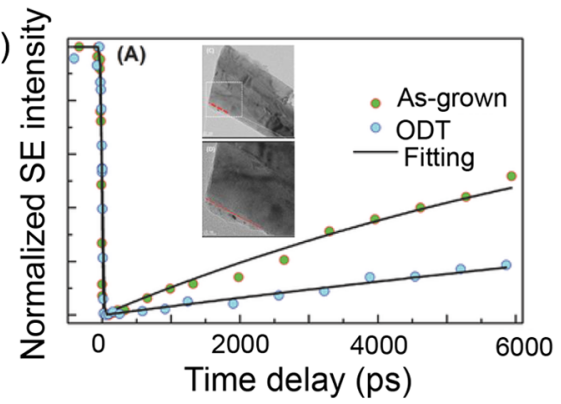

Fig. 15 ODT surface passivation of InGaN/GaN NWs showing enhanced (a) photoluminescence and (b) EQE. Reproduced with permission from Ref. 64. (c) Schematic of 4D S-UEM set-up schematic and (d) SE intensity improvement for the ODT-passivated InGaN NWs. The inset shows the SEM image of carbon deposition at the NW sidewall. Reproduced with permission from Refs. 141 and 142.

technique for direct visualization of the material surface dynamics at high spatial and temporal resolution. Figure 15(c) shows the 4-D S-UEM schematic used for analyzing the above-mentioned NWs after surface treatment. A femtosecond laser and electron pulses are synchronized to generate photoelectron wave packets that initiate the NWs carrier dynamics. Collecting the emitted secondary electrons (SEs), we analyzed the time regimes between the electron and the optical pulses and were able to directly visualize the surface recombination processes.

Figure 15(d) shows the kinetics of the time-resolved SE intensities of the InGaN NWs. As-grown NWs showed a faster recovery of the SE intensity, signifying that the SE are less interfered with by the generated excess carrier, which easily recombines through the additional sidewall leakage path. ${ }^{142}$ Once the sidewalls are passivated using ODT, the absence of the additional leakage path led to a slower recovery of SE.

Similarly, attaining a quantitative understanding for AlGaN nanostructures, particularly on the recombination mechanisms and surface states, is required. Therefore, research opportunities still exist in extending the above-mentioned techniques (4D S-UEM, DLOS, and DLTS) for AlGaN-NWs devices or even other ultrawide-bandgap materials and nanostructures. Similar to ODT, 1,2-ethanedithiol, a shorter carbon-chain organic-thiol compound, has been used to treat group-III nitride semiconductors for improved characteristics, such as in the treatment of InGaN NW surfaces for improved photoelectrochemical water splitting performance. ${ }^{145,146}$

Other instances of semiconductor surface-treatment studies are summarized in the following. Mi's group used ammonium sulfide to increase the quantum efficiency of the InGaN-based white-light NWs-LEDs in an effort to reduce the nonradiative recombination centers. ${ }^{147}$ The surface states' passivation in NWs has also been reported in GaAs, ${ }^{148} \operatorname{InAs},{ }^{149}$ and $\operatorname{InP}{ }^{150}$ as it replaces the surface oxide while creating an In-S bond, hence reducing the mid-gap states. 
Also, by treating AlGaN-based UV NWs-LED using a KOH solution over a 40-s period, we observed an improvement in PL intensity by $80 \%$ compared with an untreated sample. In this case, it is very likely that the improvement is associated with both the etching of the p-GaN contact layer and the top-surface oxide removal. A fabricated device after such treatment showed improvement in the turn-on voltage, reduction in contact resistivity, and $\sim 50 \%$ enhancement in output power. ${ }^{47}$

Other than passivating the surface states by employing chemical treatments or depositing dielectric materials, researchers achieved in situ passivation by spontaneously growing shell layers around semiconductor NWs, in general. For example, AlGaAs shells were intentionally grown around GaAs NWs to prevent subsequent oxidation upon exposure to the atmosphere, without which recombination velocities would increase and PL emission would reduce. ${ }^{151}$ Similarly, AlInP core-shells were grown to protect and passivate GaAs NWs. Further device studies by fitting and modeling the current-voltage characteristics showed improvement in surface recombination velocity and reduction in surface charge trap density. ${ }^{152}$ In group-III nitride materials, such as $\mathrm{AlGaN}$, due to the short diffusion length of $\mathrm{Al}$ compared with $\mathrm{Ga}$, $\mathrm{Al}$ adatoms have a strong tendency to accumulate at the NW sidewalls, thus resulting in the formation of an Al-rich AlGaN shell. ${ }^{67,153}$ This leads to strong carrier confinement at the surface of the NW, reducing the nonradiative recombination processes and increasing the overall device performance in LEDs ${ }^{30,154}$ and lasers. ${ }^{126}$ When comparing GaN and AlGaN/GaN core-shell NWs using DLOS, a deep level in GaN NWs at $E_{v}+2.6 \mathrm{eV}$ was measured, and this was ascribed to the surface state as it was not observed in the $\mathrm{AlGaN} / \mathrm{GaN}$ core-shell NWs. ${ }^{143}$ Nguyen et al. ${ }^{155}$ demonstrated an 10× photoluminescence intensity improvement in AlGaN core-shell NWs as well as an increase in carrier lifetime, suggesting the reduced nonradiative recombination rate as

(a)
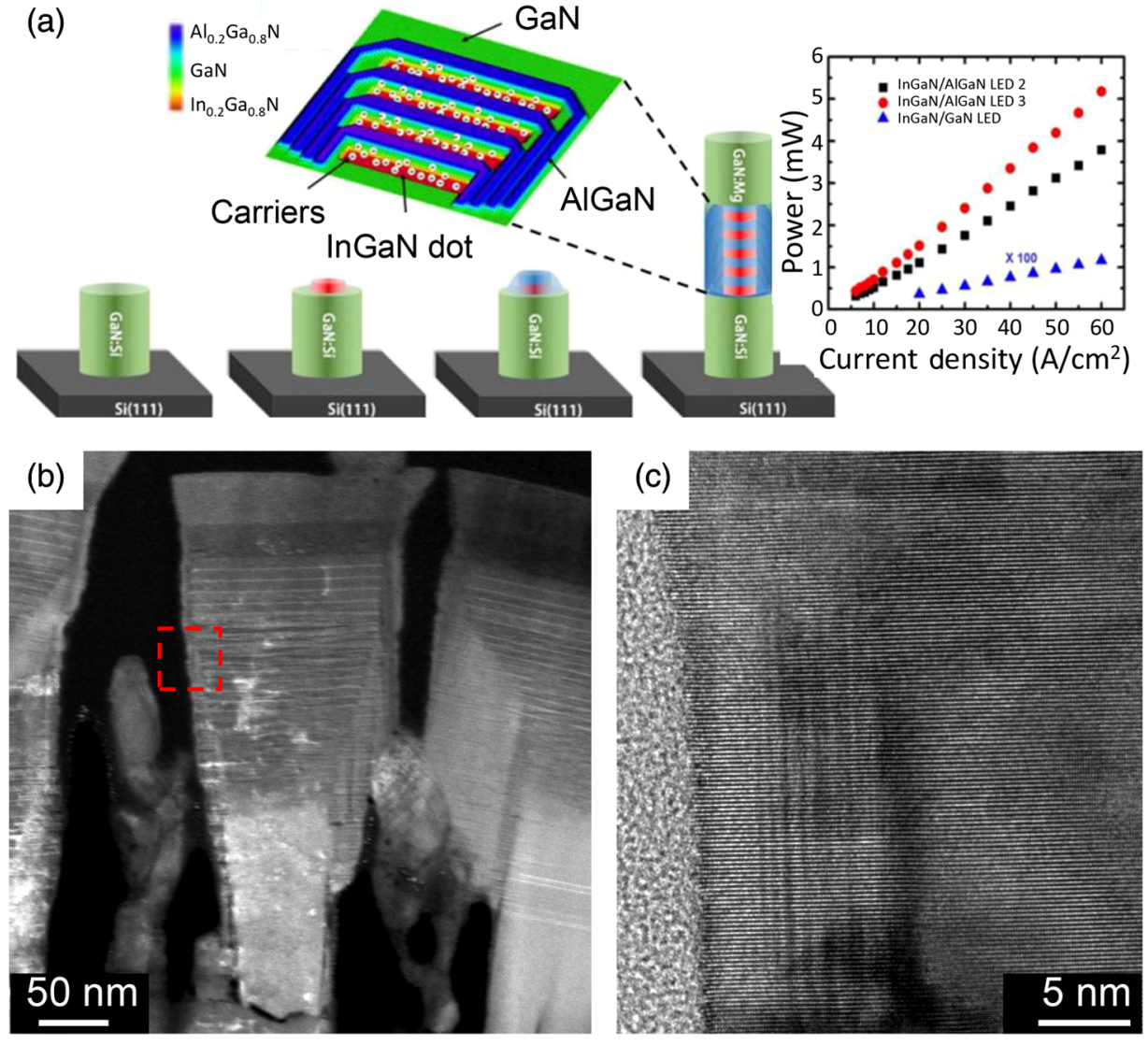

Fig. 16 (a) InGaN/GaN growth schematic with AIGaN core-shell layer and output power enhancement. Reproduced with permission from Ref. 155. (b) HAADF-STEM of AIGaN-based p-i-n NW structures on $\mathrm{Ti}(500 \mathrm{~nm}) / \mathrm{Mo}$ substrate. (c) Active region showing multiple AIGaN shells surrounding 15 stacks of uniform quantum disks/quantum barriers. 
shown in Fig. 16(a). Jiang et al. ${ }^{156}$ also demonstrated the improvement of the minority carrier lifetime by increasing the AlGaN core-shell thickness. A detailed HAADF-STEM image of the AlGaN shell is also shown in Fig. 16(b), and it clearly indicates that the NWs were surrounded by AlGaN shells. At high magnification, the shell layer around the active region was found to consist of multiple-stacks of shells as shown in Fig. 16(c).

\section{UV-Spectrum Nanowires-LED Characterization by CAFM and Reliability Test, and Solar-Blind Photodetector}

This section discusses several important characterization techniques that are closely relevant to eventual light emitter implementation. Further, the use of NW for solar-blind photodetection is presented.

\subsection{Nanoscale Electrical Characterization by cAFM}

Due to the statistical nature of self-assembled NWs growth and partly related to variation in growth parameters, the morphology and structural properties of NWs require dedicated optical and electrical characterization. For instance, Brandt et al. ${ }^{157}$ established a method to characterize the degree of coalescence of GaN NWs by applying an area-perimeter relationship of cross-sectional shape. This is primarily a study of the surface morphology of the NWs using SEM at an acceleration voltage of $5 \mathrm{kV}$ and a magnification of $20,000 \times$. Several hundreds of NWs were measured over a $30-\mu \mathrm{m}^{2}$ area and then individually analyzed for quantitative determination of the degree of coalescence. By this means, a method to perform the nanoscale structural characterization was established to determine the dominant coalescence mechanism of NWs ensemble. Moreover, as growth proceeds, the eventual nucleation of dislocations at the NWs coalescence boundaries forms the nonradiative recombination centers that affect the ultimate device performance. On the other hand, Pfüller et al. ${ }^{158}$ carried out nanoscale optical characterization by studying the low-temperature photoluminescence spectrum of an individual NW over an area of $200 \times 200 \mu \mathrm{m}^{2}$ and reported a large variation in luminescence between single NWs.

Limbach et al. ${ }^{159}$ analyzed the electroluminescence emission from InGaN NWs-LEDs and observed that only a few NWs achieve enough current injection for light emission. This was attributed to the spatial inhomogeneity in the NWs and the fluctuation in series resistance. Jahangir et al. ${ }^{160}$ further examined the effect of NWs coalescence with fabricated InGaNbased NWs-LEDs. They revealed that, due to the coalescence of NWs, defects are produced at the boundaries and a significant fraction of the NWs was not illuminating under current injection. Apparently, such observation calls for further nanoscale electrical characterization, and this is well offered by the cAFM technique.

The cAFM technique is a powerful technique that enables the simultaneous batch characterization of individual NWs for their surface topography and current distribution at the nanometer level. It can be considered a potential step for rapid inspection of the electrical properties before a full-fledge device fabrication. The conductive tip, being smaller than the NWs diameter, enables high-resolution measurement of individual NWs and large area statistical mapping. The technique has previously been explored in NWs solar cells, ${ }^{161,162}$ in nanogenerators, ${ }^{163-166}$ and only recently on group-III nitride AlGaN-based UV NWs-LEDs.

Myers's group reported the nonuniformity study of a graded AlGaN single NW and an area of NWs both with tunnel junction incorporated. ${ }^{167}$ Their studies showed the improved electrical injection and reduced turn-on voltage compared with devices without the tunnel junction. At the nanoscale level (single NW), such tunnel junction integration enables an increase in current injection through the single NW. Approximately 100 individual NWs and $\sim 20$ NWs-devices with an area of $300 \times 300 \mu \mathrm{m}^{2}$ were tested. Figure 17(a) shows the current-voltage curves of both single and full NWs device, whereas Figs. 17(b)-17(d) compare the nanoscale and microscale turn-on or threshold voltages ( $V_{\text {th_nano }}$ or $V_{\text {th_macro }}$ ), differential conductance $\left(G_{\text {nano }}\right.$ or $\left.G_{\text {macro }}\right)$, and leakage current ( $I_{\text {leak_nano }}$ or $\left.I_{\text {leak_macro }}\right)$. The same group further analyzed the current spreading on NWs-LEDs and the mechanism that reduces the electroluminescence emission also by employing cAFM. ${ }^{168}$ Due to the presence of electrical shorts (low-resistance 
(a)

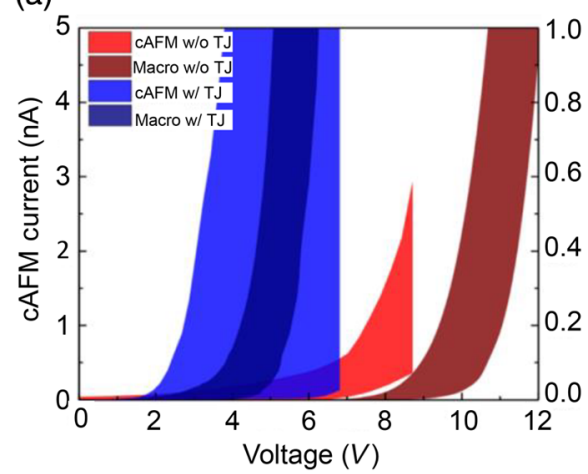

(b)

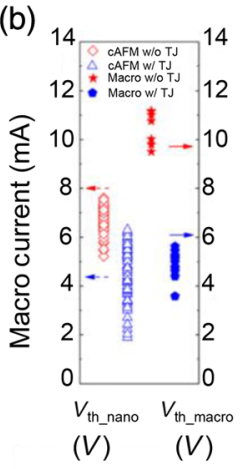

(c)

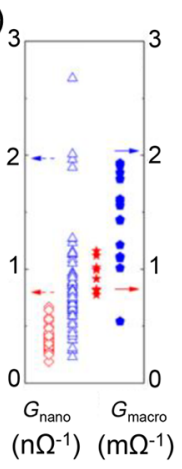

(d)

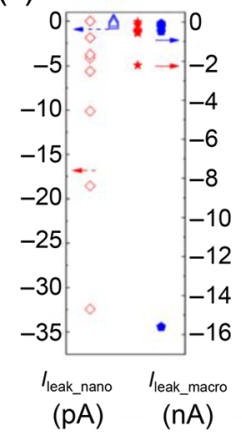

Fig. 17 cAFM measurement for graded AIGaN NWs: (a) current versus voltage curves, (b) comparison of nanoscale and macroscale turn-on or threshold voltages $\left(V_{\text {th_nano }}\right.$ or $\left.V_{\text {th_macro }}\right)$, (c) comparison of nanoscale and macroscale differential conductance $\left(G_{\text {nano }}\right.$ or $\left.G_{\text {macro }}\right)$, and (d) leakage current at $-1 \vee\left(I_{\text {leak_nano }}\right.$ or $\left.I_{\text {leak_macro }}\right)$. Reproduced with permission from Ref. 167.

NWs) where most of the current passes through, uniform current distribution was not possible. Taking this into account, the ensemble NWs-LED were intentionally overloaded with relatively high voltage to render them as open circuit paths, thereby allowing uniform distribution of current across the whole device. This leads to 33\% enhancement of peak electroluminescence and reduction in leakage current. Thus cAFM measurement results clearly revealed a way for quantifying nonuniform current injection in NWs for a rapid nanoscale electrical characterization prior to fabrication of NWs-based devices.

\subsection{Reliability of AlGaN Nanowires-LEDs}

Over the past years, the efficiency of group-III nitride AlGaN-based LEDs has improved tremendously. As mentioned in the previous Secs. 3 and 6, AlGaN materials are robust and chemically inert and possess a relatively long lifetime. These properties make them suitable for substituting mercury lamps that degrade after the effective working lifetime. Despite GaNbased LEDs having commercially proven lifetimes in the order of $10^{5} \mathrm{~h}$, the possible degradation mechanism includes the increase in nonradiative recombination centers in the active region, the increase in junction temperature at elevated current injection, the increase in resistivity due to the modification of the metal contacts, the formation of hydrogen complexes, and the degradation of the package epoxy and plastic material. All of these degradation mechanisms have been thoroughly covered in InGaN/GaN visible LEDs and lasers, ${ }^{169-177}$ and detailed reviews have been published elsewhere. ${ }^{178-180}$

As for UV devices, the lifetime is still limited due to the high dislocation density, high-dopant ionization efficiency, and high Joule heating. To date, there are only a few reports on planar AlGaN-based device stability and degradation mechanisms. ${ }^{181,182}$ Meneghini et al. ${ }^{183}$ analyzed AlGaN planar LEDs emitting at a 280- and 295-nm stress test under DC bias, and they attributed the device degradation to the generation of nonradiative recombination centers, especially at low-injection current. Moreover, tunneling effects and charge distribution in the active region contributed to the overall optical power degradation. Monti et al. ${ }^{184}$ reported a UV-B Al(In)GaNbased LED stability test at a high-injection current of $350 \mathrm{~A} / \mathrm{cm}^{2}$. They noticed a decrease in the emission peak intensity after $50 \mathrm{~h}$ of stress that was related to the escape of carries from the wells and the diffusion of point defects in the active region, respectively. UV-C AlGaN-based LEDs degradation was also tested by Glaab et al. ${ }^{185}$ and showed a reduction in optical power by $\sim 40 \%$ after $250 \mathrm{~h}$ of operation with a decrease in the overall device capacitance. While the former is mainly attributed to the generation of acceptor-like defects, the latter was attributed to the reduced charges in the space-charge regions. A calculation showed that this region increased by $10 \mathrm{~nm}$ after stress.

Group-III nitride UV NWs technology is very much at its infancy compared with the planar counterpart, and therefore, lower efficiencies were often reported, as discussed in the Secs. 1 and 6. 
However, to advance the research into eventual practical applications, understanding the degradation mechanisms is of particular importance. In this regard, we recently reported the softstress analysis of $\mathrm{AlGaN} / \mathrm{GaN}$ quantum-confined NWs-LED emitting at $\sim 340 \mathrm{~nm}$, under DC bias. Figure 18(a) shows the output power and voltage versus stress time at $16 \mathrm{~A} / \mathrm{cm}^{2}{ }^{48}$ Despite a drop in voltage after $5 \mathrm{~h}$, likely due to a rise in junction temperature, the power remains relatively stable. However, in-depth analysis and curves fitting by employing the LED rate equation resulted in showing degradation in the active region.

Moreover, we studied the low- and high-injection current regimes before and after the stress test to yield the corresponding slopes. Figure 18(b) shows the quadratic and linear behavior regimes of the optical power after a $5 \mathrm{~h}$ soft stress test. When comparing the curves before

(a)

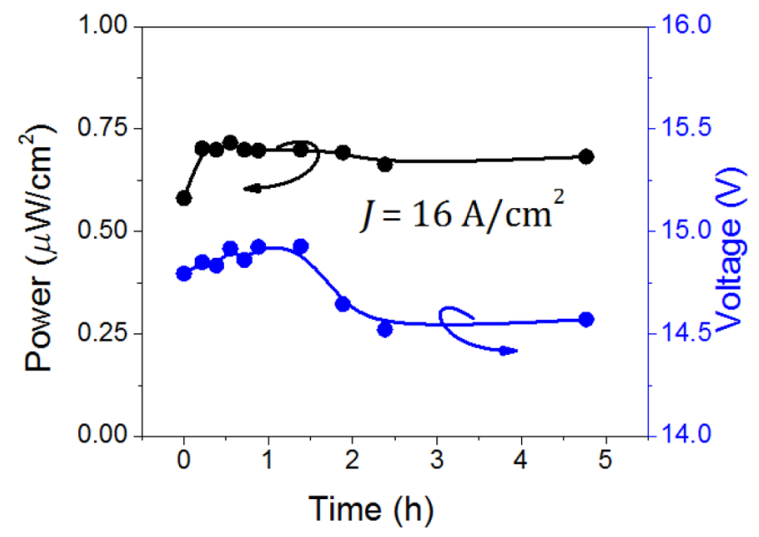

(b)

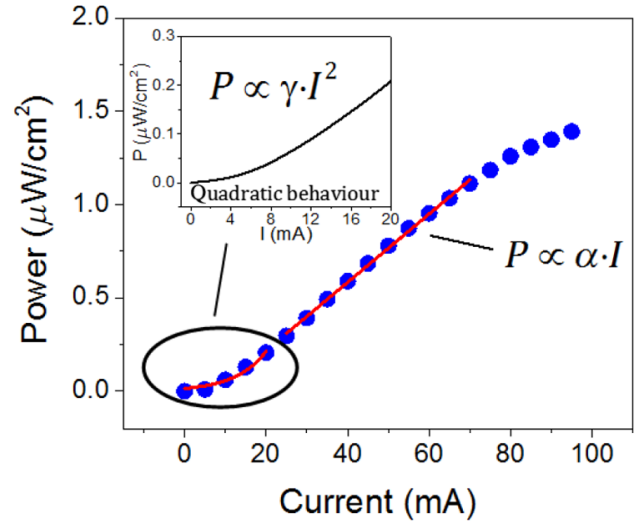

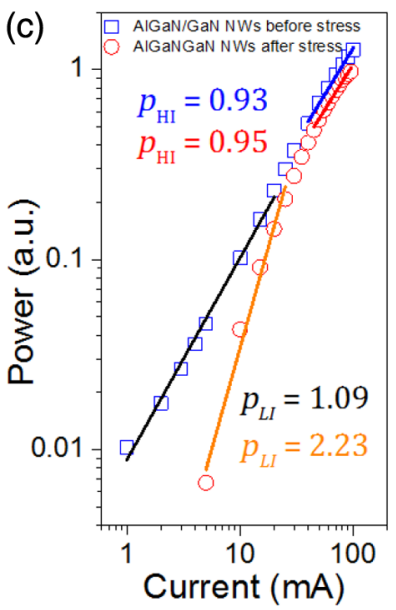

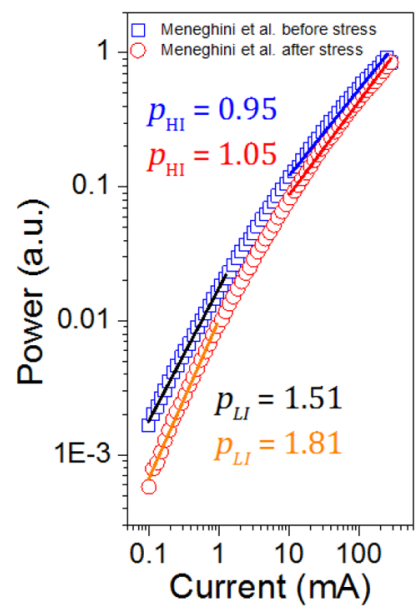

Fig. 18 AIGaN/GaN NWs-LED reliability test. (a) Power ( $Y$-axis) and voltage (alternate $Y$-axis) versus time plot at low-injection current. (b) Power-current characteristic of the device after stress test showing the low- and high-injection current regimes. (c) Semilog plot of power versus current comparing characteristics of NWs and planar devices. Reproduced with permission from Ref. 48. 
and after the stress test, we noticed a change at low-injection currents. To explain this, we extracted the slopes of the semilog power-current curves [Fig. 18(c)]. At a high-injection current, the sample before the stress test shows a slope $\left(P_{\mathrm{HI}}\right)$ of 0.93 , whereas after stress, a value of 0.95 was obtained. The value of $\sim 1$ confirms the dominance of the radiative recombination rates as indicated by the LED rate equation simplified for high-injection currents. ${ }^{48}$ On the other hand, at low-injection currents, the slopes of the sample before and after the stress test $\left(P_{\mathrm{LI}}\right)$ are 1.09 and 2.23 , respectively. We can then deduce that the nonradiative recombination processes dominated in the sample after stress (slope $\sim 2$ ), implying that degradation has occurred. It can be concluded that nonradiative processes dominate after stressing the device in both regimes, although it is more prominent at low injections. In fact, at high current, the number of nonradiative recombination centers tends to saturate, leading to a reduced impact on the optical power output, as previously reported in AlGaN planar LED. ${ }^{186}$ We then compared our results with the GaN-based planar UV LEDs. ${ }^{174}$ Figure 18(c) shows a larger slope of 1.51 (GaN-based planar UV LED) compared with 1.09 for the NWs-device before stress. This suggests larger nonradiative recombination at low-injection current for the planar UV device, hence confirming the higher crystal quality of the as-grown NWs. However, after the stress test, the NWs-device shows a slope of 2.23, larger than the one of the GaN-based planar UV LED (1.81), meaning that the former is affected by higher nonradiative recombination centers that induce a faster device degradation.

As mentioned above, junction temperature affects the device reliability. In particular, for $\mathrm{AlGaN}$ materials, where the thermal heating is more prominent, the optical power and the efficiency are highly affected by the junction temperature. UV GaN and AlGaN planar LEDs junction temperatures have been reported, ${ }^{187,188}$ but, to date, there are no such reports for UV NWs-LEDs.

\subsection{Solar-Blind AlGaN-Based Nanowires-Photodetectors}

The fast development in UV semiconductors has attracted attention for devices beyond LEDs development. Astronomy, fire detection, solar light monitoring, communication, and missiles detection are some applications for UV photodetectors. The main devices employed for UV detection are the photomultiplier tubes (PMTs), thermal detectors, charge-coupled devices (CCDs), and semiconductor photodetectors. However, PMTs have a large volume and high fragility, while thermal detectors and CCDs show low speed and wavelength-independent response. Contrariwise, semiconductor photodiodes are small and low-cost devices with good linearity and sensitivity for high-speed application. Although the most common UV photodetectors are silicon-based, these small-bandgap devices suffer from high-energy radiation degradation problems, high sensitivity to visible and IR radiation, low directivity, and costly optical filters necessary for wavelength selectivity. AlGaN-based photodetectors can overcome these problems as they have better spectral selectivity due to the direct bandgap and capability of tuning the cut-off wavelength by varying the composition of the ternary alloys. ${ }^{189}$

Since the first demonstration of GaN-based UV photodetectors by Khan et al. ${ }^{190}$ the GaN and $\mathrm{AlGaN}$ photodetectors have shown responsivity and $\mathrm{EQE}$ of 0.1 and $0.8 \mathrm{~A} \cdot \mathrm{W}^{-1}$ as well as 30 to 40 and $25 \%$ (depending on the wavelength), respectively. ${ }^{191} \mathrm{~A}$ dark current of $8.2 \times 10^{-11} \mathrm{~A} / \mathrm{cm}^{2}$ (Ref. 192) and breakdown voltage of up to $200 \mathrm{~V}$ have also been reported for deep-UV AlGaN-based devices. ${ }^{193}$ However, the current planar AlGaN-based photodetector devices still suffer from low crystal quality caused by the lack of lattice-matched substrates, high-dopant activation energy, and the polarization field. Unlike InGaN/GaN structures, where the built-in electric field is mainly caused by the difference in piezoelectric polarizations of the materials, in $\mathrm{AlGaN} / \mathrm{GaN}$ structures, both piezoelectric and spontaneous polarizations play a crucial role. In fact, the latter is dependent on both wells and barrier width. Lefebvre et al. reported a systematic study on $\mathrm{AlGaN} / \mathrm{GaN}$ thickness-dependent wells and barriers. In general, the thinner the well, the less pronounced the internal field, hence lower band bending. They proved that, for the barrier as thin as $5 \mathrm{~nm}$, carriers tend to escape (in the form of nonradiative recombination processes) from thinner wells to thicker wells. ${ }^{110}$ An internal field as high as $0.73 \mathrm{MV} / \mathrm{cm}$ was estimated by Deguchi et al. ${ }^{194}$ on an AlGaN/GaN/AlGaN single quantum well structure, showing low-emission efficiency. Such fields can be screened by high-free-carrier 
concentration that can be introduced either by injection current or by doping, the latter being advantageous when utilizing NWs.

In this regard, NWs photodetectors have been studied and demonstrated with remarkable results. Other than improved material properties, NWs allow device miniaturization due to their small diameter and increased light trapping for enhanced light absorption. In fact, enhanced absorptance was studied for optical modes parallel to the NWs axis, exceeding that of planar structures. ${ }^{195}$ The refractive index contrast also plays a crucial role in the modal resonance absorption, causing the optical absorption to be higher than thin film structures. ${ }^{196}$ Moreover, NW photodetectors showed superior sensitivity compared with the planar devices, due to the high surface-to-volume ratio that leads to larger photoconductive gain. ${ }^{197}$ Among the wide-bandgap materials, $\mathrm{ZnO} \mathrm{NWs}$ with a room temperature bandgap of $3.37 \mathrm{eV}$ have been widely studied for UV photodetectors and have shown high-photoconductive responses upon exposure to UV radiation. ${ }^{198-201}$ Responsivity as high as $10^{3} \mathrm{~A} / \mathrm{W}$ has been reported on $\mathrm{n}-\mathrm{i}-\mathrm{p} \mathrm{ZnO}$ NWs grown on a GaN template. ${ }^{202}$ Single $\mathrm{ZnO}$ NW photodetectors have also been demonstrated. The high-photoconductive gain of $10^{8}$ was achieved and an issue related to oxygen surface states was addressed, which increases the photocurrent lifetime and the short transit time that results from the low dimensionality of the NWs s structure. ${ }^{203}$ Despite the achievement, $\mathrm{ZnO}$-based materials showed an uncontrollable emission wavelength as a result of unstable p-type $\mathrm{ZnO}$ as well as the lack of carrier confinement.

GaN-based NWs-photodetectors have emerged as a stable device with visible- and solarblind properties. Compared with Si-based and carbon nanotubes, III-V NW semiconductors have a larger carrier concentration and mobility that are fundamental for high-speed devices and a cut-off wavelength that can be adjusted from 365 to $210 \mathrm{~nm}$. A single GaN NW photodetector was reported showing large UV-to-visible responsivity ratio ${ }^{204,205}$ [Figs. 19(a)-19(c)] and high responsivity of $\sim 10^{4} \mathrm{~A} / \mathrm{W}$ along with fast response speed. ${ }^{206}$ Figure 19(d) shows the GaN NWs ensemble device with responsibility peak of $25 \mathrm{~A} / \mathrm{W}$ at $357 \mathrm{~nm} .{ }^{207}$ MOCVD-grown solar-blind AlGaN-based UV-C NWs-photodetectors showed responsivity peaks as high as $0.115 \mathrm{~A} / \mathrm{W}^{208}$ and $0.72 \mathrm{~A} / \mathrm{W}$ for AlGaN nanoflowers [Figs. 19(e) and 19(f)]. ${ }^{209}$

To this end, AlGaN-based NWs-photodetectors were grown on metal thin film ${ }^{42}$ and measured using the responsivity measurement setup shown in Fig. 20(a). The UV light was generated by a xenon-mercury arc lamp, filtered by Newport monochromator, and focused on the sample stage with the illumination area of $3.5 \mathrm{~mm} \times 5.5 \mathrm{~mm}$. The wavelength of incident light can be tuned from $240 \mathrm{~nm}$ to the visible spectrum. The dark current and photogenerated current are collected by the Keithley 4200 semiconductor characterization system. The sample structure of the AlGaN-based NWs having embedded multiple quantum disk (AlGaN MQdisks) is also shown in Fig. 20(b). The photocurrent versus voltage plot of the NWs-photodetector as a function of excitation wavelength is shown in Fig. 20(c). It can be noticed that the dark current (in black) is relatively small, especially at increased voltage values, reaching $5 \times 10^{-9} \mathrm{~A}$ at $-4 \mathrm{~V}$. The photocurrent is measured at different excitation wavelengths at $\sim 0.5 \mathrm{~mW} / \mathrm{cm}^{2}$, and larger values are obtained at a shorter excitation wavelength of $265 \mathrm{~nm}$. As the AlGaN-based NWs-device shows an electroluminescence peak at $325 \mathrm{~nm}$ when forward biased [Fig. 20(c)], the $I-V$ curves above bandgap (at 330 and $400 \mathrm{~nm}$ ) show negligible photocurrent. The inset shows the reverse and forward bias $I-V$ curves of the NWs-device.

The performance of a photodetector is mainly compared by measuring the responsivity $R$ that can be expressed as

$$
R=\frac{I_{\mathrm{ph}}}{P_{\mathrm{inc}}},
$$

where $I_{\mathrm{ph}}$ is the photocurrent and $P_{\text {inc }}$ is the incident optical power. The AlGaN-based NWsphotodetector responsivity versus wavelength at different biases is presented in Fig. 20(d). The main peak at $286 \mathrm{~nm}$ is ascribed to the absorption at the high $\mathrm{Al}$ composition barrier. In fact, the photoluminescence spectra at 10 and $300 \mathrm{~K}$ show a similar peak. The other longer wavelength peaks at $\sim 320$ and $\sim 360 \mathrm{~nm}$ are attributed to the quantum disks and GaN nucleation layer emissions, respectively. The sensitivity of the device is calculated as ${ }^{210}$ 

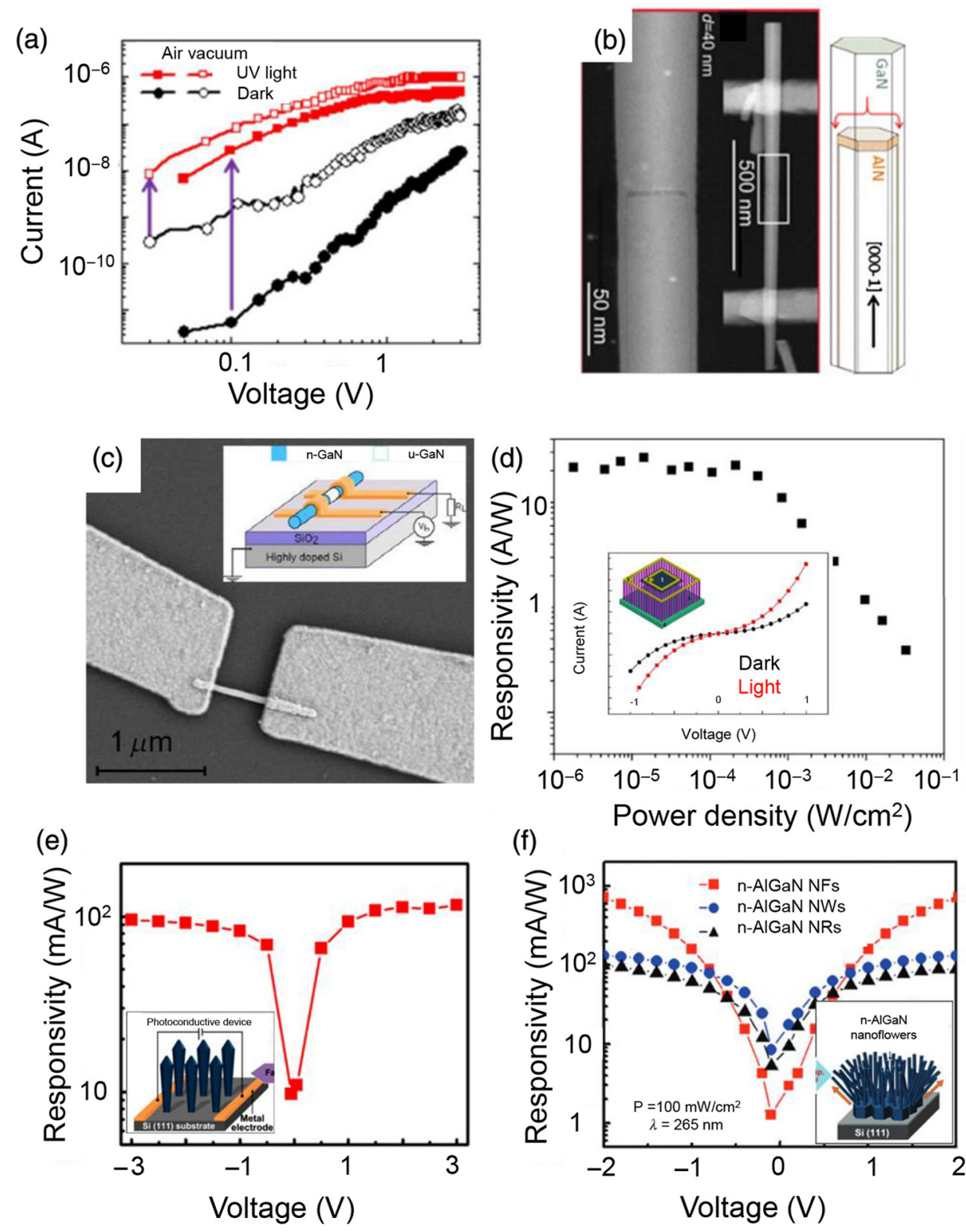

Fig. 19 (a) Photocurrent in single GaN/AIN/GaN NW and (b) schematic of the NW structure. Reproduced with permission from Ref. 205. (c) SEM image of the single GaN NW and contact pads. The inset shows the schematic of the device. Reproduced with permission from Ref. 204. (d) The responsivity of UV GaN NW. The inset shows the schematic and $I-V$ characteristics under dark and illumination condition. Reproduced with permission from Ref. 207. (e) The responsivity of AIGaN nanorods and the respective inset shows device schematic. Reproduced with permission from Ref. 208. (f) The responsivity of AIGaN nanoflowers, nanorods, and NWs with an inset showing a device schematic. Reproduced with permission from Ref. 209.

$$
S=\frac{I_{\mathrm{ph}}}{I_{\mathrm{dark}}},
$$

where $I_{\mathrm{ph}}$ and $I_{\mathrm{dark}}$ are the photocurrent and the dark current, respectively. At $-4 \mathrm{~V}, S$ has a value of $3.81 \times 10^{2}$.

The responsivity value, however, is still lower than previously reported AlGaN nanostructures ${ }^{208,209}$ and AlGaN thin film photodetectors. ${ }^{193,211}$ We emphasize that the device optimization has not been conducted and a relatively lower voltage was applied compared with that of AlGaN-based planar photodetectors. Therefore, there is still room for large improvement, 
(a)
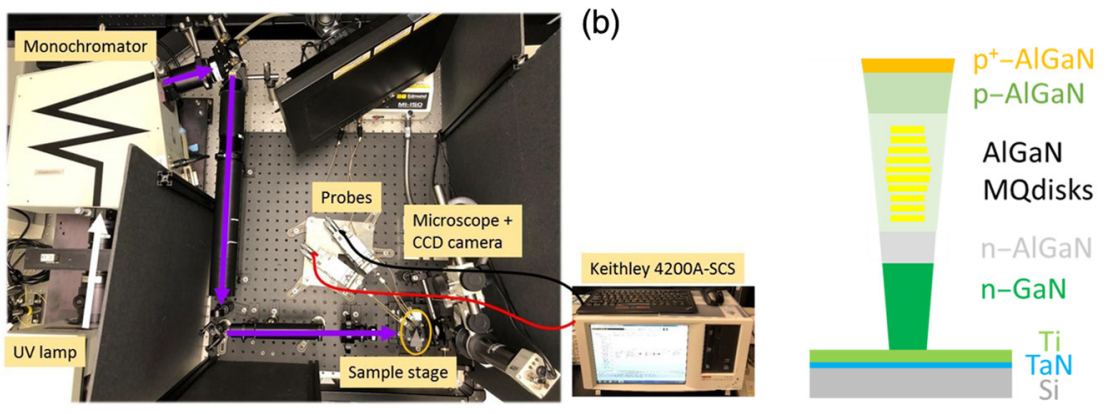

(c)

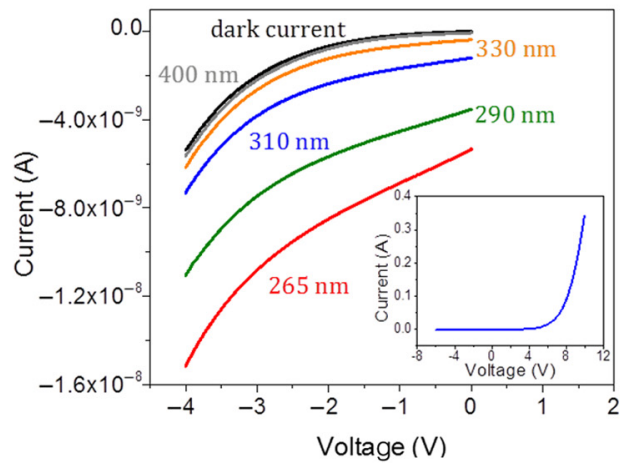

(d) $\bar{\xi}$

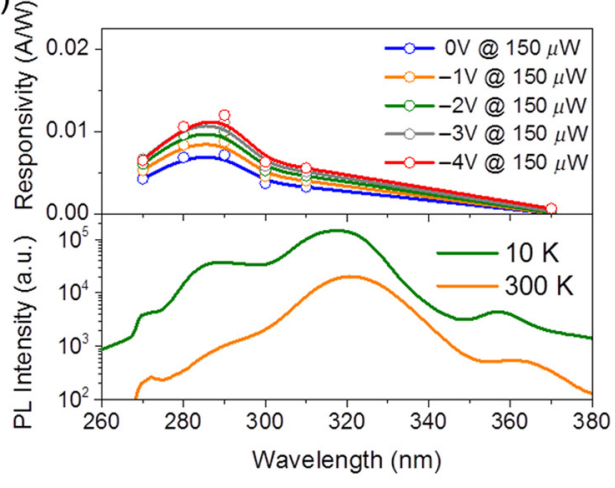

Fig. 20 (a) The setup for responsivity measurement. (b) The structure of AlGaN-based NWs grown on $\mathrm{Ti} / \mathrm{TaN} / \mathrm{Si}$. (c) Reverse bias current versus voltage curves at the different excitation wavelengths. The inset shows the forward bias current versus voltage curve. (d) Responsivity spectra with $0.5 \mathrm{~mW} / \mathrm{cm}^{2}$ lamp excitation and photoluminescence spectra.

given the technology infancy of AlGaN-based NWs-photodetectors. In fact, such nanostructures show the dislocation-free active region that not only allows improved photocurrent but enables the NWs growth on low-cost and large size silicon substrates. Moreover, as described in Sec. 6, the spontaneous AlGaN passivation at the NW sidewalls is believed to reduce the SRH nonradiative recombination, hence lowering the device leakage current for improved photodetection efficiency. Therefore, the demonstration of NWs-devices beyond LEDs development, such as photodetectors, constitutes an important step for advancing UV-spectrum semiconductor research toward self-assembled NWs for solar-blind photodetectors.

\section{Conclusions}

This paper provides an outlook on the current development of PA-MBE grown UV AlGaN-based NWs growth, nanoscale characterization, and devices implementation. Specifically, we showcase several key enabling techniques for the self-assembled NWs technology, which offer insights on the prospects of the PA-MBE technology moving forward. A key highlight of the report was on the importance of growing these nanostructures on diffusion-barrier-metal/ silicon template substrates for large current handling and heat management, which are issues faced by the group-III nitride NWs community when growing NWs on bare silicon substrates. Moreover, the use of foundry-compatible Ti/TaN thin film on scalable Si substrate demonstrates the practicality of our approach. We further emphasize the significant advantage of employing nanostructure as a niche solution to mitigate the effects of piezoelectric polarization fields and lattice matching requirements for eventual high-performance light-emitting devices. Furthermore, the need to effectively treat the NWs surface states and develop surface treatment solutions were highlighted, along with the use of 4D S-UEM technique to directly monitor the surface carrier dynamics before and after surface treatment. We further identified a recently employed cAFM technique as a critical step for in-process probing at nano- and macroscale prior to device fabrication. Finally, we analyzed the degradation mechanism of AlGaN-based 
UV NWs LED by means of a direct-current stress test and demonstrate a UV-spectrum AlGaNbased NWs-photodetector. Indeed, the research opportunities for these nanostructures are still boundless.

\section{Acknowledgments}

We acknowledge the financial support from the King Abdulaziz City for Science and Technology (KACST) under Grant No. KACST TIC R2-FP-008. This work was partially supported by the King Abdullah University of Science and Technology (KAUST) baseline funding No. BAS/1/1614-01-01 and MBE equipment funding No. C/M-20000-12-001-77.

\section{References}

1. T. Mukai et al., "High-power UV InGaN/AlGaN double-heterostructure LEDs," J. Cryst. Growth 15(189-190), 778-781 (1998).

2. S. Nakamura, "The roles of structural imperfections in InGaN-based blue light-emitting diodes and laser diodes," Science 281(5379), 956-961 (1998).

3. Y. Sun et al., "Room-temperature continuous-wave electrically injected InGaN-based laser directly grown on Si," Nat. Photonics 10(9), 595-599 (2016).

4. V. Adivarahan et al., "Robust $290 \mathrm{~nm}$ emission light emitting diodes over pulsed laterally overgrown AlN," Jpn. J. Appl. Phys. 46(36) L877-L879 (2007).

5. V. Y. Davydov et al., "Absorption and emission of hexagonal InN. Evidence of narrow fundamental band gap," Phys. Status Solidi Basic Res. 229(3), R1-R3 (2002).

6. J. Kuyyalil et al., "Effect of interfacial lattice mismatch on bulk carrier concentration and band gap of InN," J. Appl. Phys. 112, 083521 (2012).

7. S. Hwang et al., "276 nm substrate-free flip-chip AlGaN light-emitting diodes," Appl. Phys. Express 4(3), 032102 (2011).

8. H. Hirayama et al., "222-282 nm AlGaN and InAlGaN-based deep-UV LEDs fabricated on high-quality AIN on sapphire," Phys. Status Solidi Appl. Mater. Sci. 206(6), 1176-1182 (2009).

9. S. Vilhunen et al., "Ultraviolet light-emitting diodes in water disinfection," Environ. Sci. Pollut. Res. 16(4), 439-442 (2009).

10. M. Kneissl et al., "Advances in group III-nitride-based deep UV light-emitting diode technology," Semicond. Sci. Technol. 26(1), 014036 (2011).

11. T. Kolbe et al., "(In)AlGaN deep ultraviolet light emitting diodes with optimized quantum well width,” Phys. Status Solidi Appl. Mater. Sci. 207(9), 2198-2200 (2010).

12. Y. H. Liang et al., "Progress in efficient doping of high aluminum-containing group IIInitrides," Appl. Phys. Rev. 5(1), 011107 (2018).

13. D. Jena et al., "Realization of wide electron slabs by polarization bulk doping in graded III-V nitride semiconductor alloys," Appl. Phys. Lett. 81(23), 4395-4397 (2002).

14. J. Simon et al., "Polarization-induced hole doping in semiconductor heterostructures," Science 327(5961), 60-64 (2010).

15. A. T. M. Golam Sarwar et al., "Tuning the polarization-induced free hole density in nanowires graded from GaN to AlN," Appl. Phys. Lett. 106(3), 032102 (2015).

16. J. Edmond et al., "High efficiency GaN-based LEDs and lasers on SiC," J. Cryst. Growth 272(1-4), 242-250 (2004).

17. H. Amano et al., "Metalorganic vapor phase epitaxial growth of a high quality GaN film using an AlN buffer layer," Appl. Phys. Lett. 48(5), 353-355 (1986).

18. T. D. Moustakas, T. Lei, and R. J. Molnar, "Growth of GaN by ECR-assisted MBE," Physica B 185(1-4), 36-49 (1993).

19. H. Okumura et al., "Polarity control in MBE growth of III-nitrides, and its device application," Curr. Appl. Phys. 2(4), 305-310 (2002).

20. H. Ishikawa et al., "GaN on Si substrate with AlGaN/AlN intermediate layer," Jpn. J. Appl. Phys. 38(5 Part 2), L492-L494 (1999).

21. O. H. Nam et al., "Lateral epitaxy of low defect density GaN layers via organometallic vapor phase epitaxy," Appl. Phys. Lett. 71(18), 2638-2640 (1997). 
22. E. D. Le Boulbar et al., "Design and fabrication of enhanced lateral growth for dislocation reduction in GaN using nanodashes," J. Cryst. Growth 466, 30-38 (2017).

23. C. I. H. Ashby et al., "Low-dislocation-density GaN from a single growth on a textured substrate," Appl. Phys. Lett. 77(20), 3233-3235 (2000).

24. H.-Y. Shin et al., "Reducing dislocation density in GaN films using a cone-shaped patterned sapphire substrate," J. Cryst. Growth 311(17), 4167-4170 (2009).

25. H. Hartono et al., "Reduction of threading dislocation density in GaN grown on strain relaxed nanoporous GaN template," Appl. Phys. Lett. 90(17), 171917 (2007).

26. K. Pakuła et al., "Reduction of dislocation density in heteroepitaxial GaN: role of $\mathrm{SiH}_{4}$ treatment," J. Cryst. Growth 267(1-2), 1-7 (2004).

27. M. H. Crawford, "Materials challenges of AlGaN-based UV optoelectronic devices," Semicond. Semimetals 96, 3-44 (2017).

28. H. Sekiguchi et al., "Ultraviolet GaN-based nanocolumn light-emitting diodes grown on n-(111) Si substrates by RF-plasma-assisted molecular beam epitaxy," Phys. Status Solidi Appl. Mater. Sci. 205(5), 1067-1069 (2008).

29. S. D. Carnevale et al., "Graded nanowire ultraviolet LEDs by polarization engineering," Proc. SPIE 8467, 84670L (2012).

30. Q. Wang et al., "Highly efficient, spectrally pure $340 \mathrm{~nm}$ ultraviolet emission from $\mathrm{Al}_{x} \mathrm{Ga}_{1-x} \mathrm{~N}$ nanowire based light emitting diodes," Nanotechnology 24(34), 345201 (2013).

31. T. F. Kent et al., "Deep ultraviolet emitting polarization induced nanowire light emitting diodes with $\mathrm{Al}_{x} \mathrm{Ga}_{1-x} \mathrm{~N}$ active regions," Nanotechnology 25(45), 455201 (2014).

32. A. T. M. G. Sarwar et al., "Tunnel junction enhanced nanowire ultraviolet light emitting diodes," Appl. Phys. Lett. 107(10), 101103 (2015).

33. S. Zhao et al., "Surface emitting, high efficiency near-vacuum ultraviolet light source with aluminum nitride nanowires monolithically grown on silicon," Nano Lett. 15(10), 7006-7009 (2015).

34. S. Zhao et al., "Aluminum nitride nanowire light emitting diodes: breaking the fundamental bottleneck of deep ultraviolet light sources," Sci. Rep. 5, 1-5 (2014).

35. A. G. Sarwar et al., "Semiconductor nanowire light-emitting diodes grown on metal: a direction toward large-scale fabrication of nanowire devices," Small 11(40), 5402-5408 (2015).

36. S. Zhao et al., "Sub-milliwatt AlGaN nanowire tunnel junction deep ultraviolet light emitting diodes on silicon operating at $242 \mathrm{~nm}$," Appl. Phys. Lett. 109(20), 201106 (2016).

37. S. Zhao et al., "Molecular beam epitaxy growth of Al-rich AlGaN nanowires for deep ultraviolet optoelectronics," APL Mater. 4(8), 086115 (2016).

38. B. H. Le et al., "Controlled coalescence of AlGaN nanowire arrays: an architecture for nearly dislocation-free planar ultraviolet photonic device applications," Adv. Mater. 28, 8446-8454 (2016).

39. B. J. May et al., "Nanowire LEDs grown directly on flexible metal foil," Appl. Phys. Lett. 108(14), 141103 (2016).

40. B. Janjua et al., "Self-planarized quantum-disks-in-nanowires ultraviolet-B emitters utilizing pendeo-epitaxy," Nanoscale 9(23), 7805-7813 (2017).

41. B. Janjua et al., "Droop-free $\mathrm{Al}_{x} \mathrm{Ga}_{1-x} \mathrm{~N} / \mathrm{Al}_{y} \mathrm{Ga}_{1-y} \mathrm{~N}$ quantum-disks-in-nanowires ultraviolet LED emitting at $337 \mathrm{~nm}$ on metal/silicon substrates," Opt. Express 25(2), 1381-1390 (2017).

42. D. Priante et al., "Highly uniform ultraviolet-A quantum-confined AlGaN nanowire LEDs on metal/silicon with a TaN interlayer," Opt. Mater. Express 7(12), 4214 (2017).

43. X. Liu et al., "Selective area epitaxy of AlGaN nanowire arrays across nearly the entire compositional range for deep ultraviolet photonics," Opt. Express 25(24), 30494 (2017).

44. S. M. Sadaf et al., "An AlGaN core-shell tunnel junction nanowire light-emitting diode operating in the ultraviolet-C band," Nano Lett. 17(2), 1212-1218 (2017).

45. X. Liu et al., "Molecular beam epitaxial growth and characterization of AIN nanowall deep UV light emitting diodes," Appl. Phys. Lett. 111(101), 1-4 (2017).

46. D. A. Laleyan et al., "AlN/h-BN heterostructures for $\mathrm{Mg}$ dopant-free deep ultraviolet photonics," Nano Lett. 17(6), 3738-3743 (2017). 
47. H. Sun et al., "Surface-passivated AlGaN nanowires for enhanced luminescence of ultraviolet light emitting diodes," ACS Photonics 5(3), 964-970 (2017).

48. B. Janjua et al., "Ultraviolet-A LED based on quantum-disks-in-AlGaN-nanowiresoptimization and device reliability," IEEE Photonics J. 10(2), 1-11 (2018).

49. F. Dapoigny, "Nanowire LED Innovator Aledia Announces $€ 30(\$ 36 \mathrm{M})$ Million Series-C Financing," Intel Capital Joins Existing Investors to Commercialize Certain NanowireLED Technologies for Mobile Displays, https://www.aledia.com/en/news/nanowire-ledinnovator-aledia-announces-30-million-eurosseries-c-financing-10/ (29 January 2018).

50. Y. H. Ra et al., "Full-color single nanowire pixels for projection displays," Nano Lett. 16(7), 4608-4615 (2016).

51. K. Chung et al., "Monolithic integration of individually addressable light-emitting diode color pixels," Appl. Phys. Lett. 110(11), 111103 (2017).

52. M. Yoshizawa et al., "Growth of self-organized $\mathrm{GaN}$ nanostructures on $\mathrm{Al}_{2} \mathrm{O}_{3}(0001)$ by RF-radical source molecular beam epitaxy," Jpn. J. Appl. Phys. 36(Part 2, 4B), L459-L462 (1997).

53. M. A. Sanchez-Garcia et al., "The effect of the III/V ratio and substrate temperature on the morphology and properties of GaN- and AlN-layers grown by molecular beam epitaxy on Si(111)," J. Cryst. Growth 183(1-2), 23-30 (1998).

54. B. Heying et al., "Optimization of the surface morphologies and electron mobilities in GaN grown by plasma-assisted molecular beam epitaxy," Appl. Phys. Lett. 77(18), 2885-2887 (2007).

55. S. Fernández-Garrido et al., "A growth diagram for plasma-assisted molecular beam epitaxy of GaN nanocolumns on Si(111)," J. Appl. Phys. 106(12), 126102 (2009).

56. B. Heying et al., "Control of GaN surface morphologies using plasma-assisted molecular beam epitaxy," J. Appl. Phys. 88(4), 1855-1860 (2000).

57. R. K. Debnath et al., "Mechanism of molecular beam epitaxy growth of GaN nanowires on Si(111)," Appl. Phys. Lett. 90(12), 123117 (2007).

58. J. Ristić et al., "On the mechanisms of spontaneous growth of III-nitride nanocolumns by plasma-assisted molecular beam epitaxy," J. Cryst. Growth 310(18), $4035-4045$ (2008).

59. R. Calarco et al., "Nucleation and growth of $\mathrm{GaN}$ nanowires on $\mathrm{Si}(111)$ performed by molecular beam epitaxy," Nano Lett. 7(8), 2248-2251 (2007).

60. S. D. Carnevale et al., "Mixed polarity in polarization-induced p-n junction nanowire light-emitting diodes," Nano Lett. 13(7), 3029-3035 (2013).

61. M. Tangi et al., "Bandgap measurements and the peculiar splitting of $\mathrm{E}_{2}^{\mathrm{H}}$ phonon modes of $\mathrm{In}_{x} \mathrm{Al}_{1-x} \mathrm{~N}$ nanowires grown by plasma assisted molecular beam epitaxy," J. Appl. Phys. 120(4), 045701 (2016).

62. M. Tangi et al., "Role of quantum-confined stark effect on bias dependent photoluminescence of N-polar GaN/InGaN multi-quantum disk amber light emitting diodes," J. Appl. Phys. 123(10), 105702 (2018).

63. C. Zhao et al., "Facile formation of high-quality InGaN/GaN quantum-disks-in-nanowires on bulk-metal substrates for high-power light-emitters," Nano Lett. 16(2), 1056-1063 (2016).

64. C. Zhao et al., "An enhanced surface passivation effect in InGaN/GaN disk-in-nanowire light emitting diodes for mitigating Shockley-Read-Hall recombination," Nanoscale 7(40), 16658-16665 (2015).

65. C. Himwas et al., "Alloy inhomogeneity and carrier localization in AlGaN sections and AlGaN/AlN nanodisks in nanowires with $240-350 \mathrm{~nm}$ emission," Appl. Phys. Lett. 105(24), 241908 (2014).

66. J. Ristić et al., "Characterization of $\mathrm{GaN}$ quantum discs embedded in $\mathrm{Al}_{x} \mathrm{Ga}_{1-x} \mathrm{~N}$ nanocolumns grown by molecular beam epitaxy," Phys. Rev. B: Condens. Matter 68(12), $1-5$ (2003).

67. A. Pierret et al., "Growth, structural and optical properties of AlGaN nanowires in the whole composition range," Nanotechnology 24(11), 115704 (2013).

68. B. Roul et al., "Binary group III-nitride based heterostructures: band offsets and transport properties," J. Phys. D. Appl. Phys. 48, 423001 (2015).

69. V. Consonni et al., "Nucleation and coalescence effects on the density of self-induced GaN nanowires grown by molecular beam epitaxy,” Appl. Phys. Lett. 98(7), 071913 (2011). 
70. C. Zhao et al., "Droop-free, reliable, and high-power $\mathrm{InGaN} / \mathrm{GaN}$ nanowire light-emitting diodes for monolithic metal-optoelectronics," Nano Lett. 16(7), 4616-4623 (2016).

71. S. Y. Bae et al., "Selective-area growth of vertically oriented GaN nanostructures with a hafnium pre-orienting layer," J. Cryst. Growth 468, 110-113 (2017).

72. D. van Treeck et al., "Self-assembled formation of long, thin, and uncoalesced GaN nanowires on crystalline TiN films," Nano Res. 11(1), 565-576 (2018).

73. M. M. Rozhavskaya et al., "Synthesis of GaN nano- and microwire crystals induced by a titanium nanolayer," Tech. Phys. Lett. 40(5), 372-374 (2014).

74. M. Wölz et al., "Epitaxial growth of GaN nanowires with high structural perfection on a metallic TiN film," Nano Lett. 15(6), 3743-3747 (2015).

75. E. A. Serban et al., "Magnetron sputter epitaxy of high-quality GaN nanorods on functional and cost-effective templates/substrates," Energies 10(9), 1322 (2017).

76. S. Y. Bae et al., "III-nitride core-shell nanorod array on quartz substrates," Sci. Rep. 7, 45345 (2017).

77. A. Prabaswara et al., "Direct growth of III-nitride nanowire-based yellow light-emitting diode on amorphous quartz using thin Ti interlayer," Nanoscale Res. Lett. 13, 1-9 (2018).

78. M. Ebaid et al., "Water splitting to hydrogen over epitaxially grown InGaN nanowires on a metallic titanium/silicon template: reduced interfacial transfer resistance and improved stability to hydrogen," J. Mater. Chem. A 6, 6922-6930 (2018).

79. D. Priante et al., "Ti/TaN bilayer for efficient injection and reliable AlGaN nanowires LEDs," in Conf. on Lasers and Electro-Optics (CLEO), Paper JTu2A.91 (2018).

80. K. Kishino et al., "Selective-area growth of GaN nanocolumns on titanium-mask-patterned silicon (111) substrates by RF-plasma-assisted molecular-beam epitaxy," Electron. Lett. 44(13), 819 (2008).

81. K. Kishino et al., "Improved Ti-mask selective-area growth (SAG) by rf-plasma-assisted molecular beam epitaxy demonstrating extremely uniform GaN nanocolumn arrays," J. Cryst. Growth 311(7), 2063-2068 (2009).

82. H. Sekiguchi et al., "Emission color control from blue to red with nanocolumn diameter of InGaN/GaN nanocolumn arrays grown on same substrate," Appl. Phys. Lett. 96(23), 231104 (2010).

83. G. Calabrese et al., "Molecular beam epitaxy of single-crystalline GaN nanowires on a flexible Ti foil," Appl. Phys. Lett. 108, 202101 (2016).

84. S. Fischer et al., "On p-type doping in GaN-acceptor binding energies," Appl. Phys. Lett. 67(9), 1298-1300 (1995).

85. A. T. M. G. Sarwar et al., "Ultrathin GaN quantum disk nanowire LEDs with sub-250 nm electroluminescence," Nanoscale 8(15), 8024-8032 (2016).

86. M. Kaga et al., "GaInN-based tunnel junctions in n-p-n light emitting diodes," Jpn. J. Appl. Phys. 52(8s), 08JH06 (2013).

87. K. Balakrishnan et al., "Ultraviolet light-emitting diodes based on group three nitrides," Nat. Photonics 2(2), 77-84 (2008).

88. Y. Shi et al., "Synthesis of few-layer hexagonal boron nitride thin film by chemical vapor deposition," Nano Lett. 10(10), 4134-4139 (2010).

89. A. Sajid et al., "Defect states in hexagonal boron nitride: assignments of observed properties and prediction of properties relevant to quantum computation," Phys. Rev. B 97(6), 064101 (2018).

90. Y. Taniyasu et al., "An aluminium nitride light-emitting diode with a wavelength of 210 nanometres," Nature 441(7091), 325-328 (2006).

91. H. Sekiguchi et al., "Effect of Be-doping on InGaN/GaN nanocolumn light-emitting diode structures by RF-plasma-assisted molecular-beam epitaxy," Phys. Status Solidi Curr. Top. Solid State Phys. 5(9), 3069-3072 (2008).

92. A. Kikuchi et al., "InGaN/GaN multiple quantum disk nanocolumn light-emitting diodes grown on (111) Si substrate," Jpn. J. Appl. Phys. 43(12A), L1524-L1526 (2004).

93. L. Esaki, "New phenomenon in narrow germanium junctions," Phys. Rev. 109(2), 603-604 (1958).

94. I. Ozden et al., "A dual-wavelength indium gallium nitride quantum well light emitting diode," Appl. Phys. Lett. 79(16), 2532-2534 (2001). 
95. D. C. Law et al., "Future technology pathways of terrestrial III-V multijunction solar cells for concentrator photovoltaic systems," Sol. Energy Mater. Sol. Cells 94(8), 1314-1318 (2010).

96. D. A. B. Miller et al., "Band-edge electroabsorption in quantum well structures: the quantum-confined stark effect," Phys. Rev. Lett. 53(22), 2173-2176 (1984).

97. M. R. Philip et al., "High efficiency green/yellow and red InGaN/AlGaN nanowire light-emitting diodes grown by molecular beam epitaxy," J. Sci. Adv. Mater. Devices 2(2), 150-155 (2017)

98. R. Wang et al., "High efficiency, full-color AlInGaN quaternary nanowire light emitting diodes with spontaneous core-shell structures on Si," Appl. Phys. Lett. 106(26), 261104 (2015).

99. J. Lähnemann et al., "Coexistence of quantum-confined Stark effect and localized states in an (In, Ga)N/GaN nanowire heterostructure," Phys. Rev. B 84(15), 155303 (2011).

100. J. Renard et al., "Evidence for quantum-confined Stark effect in GaN/AlN quantum dots in nanowires," Phys. Rev. B 80(12), 2-5 (2009).

101. J. H. Ryou et al., "Control of quantum-confined stark effect in InGaN-based quantum wells," IEEE J. Sel. Top. Quantum Electron. 15(4), 1080-1091 (2009).

102. T. Takeuchi et al., "Determination of piezoelectric fields in strained GaInN quantum wells using the quantum-confined Stark effect," Appl. Phys. Lett. 73(12), 1691-1693 (1998).

103. M. Leroux et al., "Quantum confined Stark effect due to built-in internal polarization fields in (Al, Ga)N/GaN quantum wells," Phys. Rev. B 58(20), R13371-R13374 (1998).

104. T. Takeuchi et al., "Quantum-confined Stark effect due to piezoelectric fields in GaInN strained quantum wells," Jpn. J. Appl. Phys. 36(Part 2, No. 4A), L382-L385 (1997).

105. H. Masui et al., "Quantum-confined Stark effect on photoluminescence and electroluminescence characteristics of InGaN-based light-emitting diodes," J. Phys. D. Appl. Phys. 41(16), 165105 (2008).

106. N. G. Young et al., "Polarization field screening in thick (0001) InGaN/GaN single quantum well light-emitting diodes," Appl. Phys. Lett. 108(6), 061105 (2016).

107. J. Lähnemann et al., "Radial Stark effect in (In, Ga)N nanowires," Nano Lett. 16(2), 917-925 (2016).

108. J. Müßener et al., "Bias-controlled optical transitions in GaN/AlN nanowire heterostructures," ACS Nano 11(9), 8758-8767 (2017).

109. R. Songmuang et al., "Strong suppression of internal electric field in GaN/AlGaN multilayer quantum dots in nanowires," Appl. Phys. Lett. 99(14), 141914 (2011).

110. P. Lefebvre et al., "Quantum-confined stark effect and recombination dynamics of spatially indirect excitons in MBE-grown GaN-AlGaN\nquantum wells," MRS Internet J. Nitride Semicond. Res. 4, 375-380 (1999).

111. N. Grandjean et al., "Self-limitation of AlGaN/GaN quantum well energy by built-in polarization field," Appl. Phys. Lett. 74(16), 2361-2363 (1999).

112. N. Grandjean et al., "Built-in electric-field effects in wurtzite AlGaN/GaN quantum wells," J. Appl. Phys. 86(7), 3714-3720 (1999).

113. H. M. Ng et al., "The effect of built-in electric field in GaN/AlGaN quantum wells with high AIN mole fraction," J. Electron. Mater. 30(3), 134-137 (2001).

114. F. Stokker-Cheregi et al., "Impact of quantum confinement and quantum confined Stark effect on biexciton binding energy in GaN/AlGaN quantum wells," Appl. Phys. Lett. 93(15), 152105 (2008)

115. S. H. Park et al., "Piezoelectric effects on electrical and optical properties of wurtzite GaN/AlGaN quantum well lasers," Appl. Phys. Lett. 72(24), 3103-3105 (1998).

116. H. M. Ng, "Molecular-beam epitaxy of $\mathrm{GaN} / \mathrm{Al}_{x} \mathrm{Ga}_{1-x} \mathrm{~N}$ multiple quantum wells on R-plane (101̄2) sapphire substrates," Appl. Phys. Lett. 80(23), 4369-4371 (2002).

117. E. Kuokstis et al., "Polarization effects in photoluminescence of C- and M-plane GaN/AlGaN multiple quantum wells," Appl. Phys. Lett. 81(22), 4130-4132 (2002).

118. Y. H. Ra et al., "Single nanowire light-emitting diodes using uniaxial and coaxial InGaN/ GaN multiple quantum wells synthesized by metal-organic chemical vapor deposition," Nano Lett. 14(3), 1537-1545 (2014). 
119. S. Park et al., "Crystal-orientation effects on the piezoelectric field and electronic properties of strained wurtzite semiconductors," Phys. Rev. B 59(7), 4725-4737 (1999).

120. C.-Y. Wang et al., "GaN nanorod light emitting diode arrays with a nearly constant electroluminescent peak wavelength,” Opt. Express 16(14), 10549-10556 (2008).

121. J. Müßener et al., "Probing the internal electric field in GaN/AlGaN nanowire heterostructures," Nano Lett. 14(9), 5118-5122 (2014).

122. Z. Mi et al., "Molecular beam epitaxial growth and characterization of $\mathrm{Al}(\mathrm{Ga}) \mathrm{N}$ nanowire deep ultraviolet light emitting diodes and lasers," J. Phys. D. Appl. Phys. 49(36), 364006 (2016).

123. S. Zhao et al., "p-type InN nanowires," Nano Lett. 13, 2-5 (2013).

124. S. D. Carnevale et al., "Polarization-induced pn diodes in wide-band-gap nanowires with ultraviolet electroluminescence," Nano Lett. 12(2), 915-920 (2012).

125. S. Zhao et al., "An electrically pumped $239 \mathrm{~nm}$ AlGaN nanowire laser operating at room temperature," Appl. Phys. Lett. 109(19), 191106 (2016).

126. K. H. Li et al., "Ultralow-threshold electrically injected AlGaN nanowire ultraviolet lasers on Si operating at low temperature," Nat. Nanotechnol. 10(2), 140-144 (2015).

127. C. Zhao et al., "Quantified hole concentration in AlGaN nanowires for high-performance ultraviolet emitters," Nanoscale, Advanced Article (2018).

128. A. Armstrong et al., "Quantitative observation and discrimination of AlGaN-and GaNrelated deep levels in $\mathrm{AlGaN} / \mathrm{GaN}$ heterostructures using capacitance deep level optical spectroscopy," Appl. Phys. Lett. 89(26), 262116 (2006).

129. A. R. Arehart et al., "Comparison of deep level incorporation in ammonia and rf-plasma assisted molecular beam epitaxy n-GaN films," Phys. Status Solidi Curr. Top. Solid State Phys. 5(6), 1750-1752 (2008).

130. A. Armstrong et al., "Characterization of majority and minority carrier deep levels in p-type GaN:Mg grown by molecular beam epitaxy using deep level optical spectroscopy," J. Appl. Phys. 103(6), 063722 (2008).

131. Z. Zhang et al., "Deep traps in nonpolar $\mathrm{m}$-plane $\mathrm{GaN}$ grown by ammonia-based molecular beam epitaxy," Appl. Phys. Lett. 100(5), 152114 (2012).

132. H. Kim et al., "Leakage current origins and passivation effect of GaN-based light emitting diodes fabricated with Ag p-contacts," Appl. Phys. Lett. 92(9), 092115 (2008).

133. T. Hashizume et al., "Chemistry and electrical properties of surfaces of $\mathrm{GaN}$ and $\mathrm{GaN} /$ AlGaN heterostructures," J. Vac. Sci. Technol. B Microelectron. Nanom. Struct. 19(4), 1675 (2001).

134. M.-H. Lo et al., "Defect selective passivation in $\mathrm{GaN}$ epitaxial growth and its application to light emitting diodes," Appl. Phys. Lett. 95(21), 211103 (2009).

135. X. Da et al., "The silicon oxynitride layer deposited at low temperature for high-brightness GaN-based light-emitting diodes," Solid. State Electron. 50(3), 508-510 (2006).

136. S. J. So et al., "Improvement of brightness with $\mathrm{Al}_{2} \mathrm{O}_{3}$ passivation layers on the surface of InGaN/GaN-based light-emitting diode chips," Thin Solid Films 516(8), 2031-2034 (2008).

137. H. Y. Liu et al., " $\mathrm{Al}_{2} \mathrm{O}_{3}$ passivation layer for $\mathrm{InGaN} / \mathrm{GaN}$ LED deposited by ultrasonic spray pyrolysis," IEEE Photonics Technol. Lett. 26(12), 1243-1246 (2014).

138. C. M. Yang et al., "Improvement in electrical and optical performances of GaN-based LED with $\mathrm{SiO}_{2} / \mathrm{Al}_{2} \mathrm{O}_{3}$ double dielectric stack layer," IEEE Electron. Device Lett. 33(4), 564-566 (2012).

139. H. Sugahara et al., "Bonding states of chemisorbed sulfur atoms on GaAs," Surf. Sci. 242(1-3), 335-340 (1991).

140. X. Wang et al., "Structural model of sulfur on GaAs(100)," J. Appl. Phys. 75(5), 2715-2717 (1994).

141. R. Bose et al., "Real-space visualization of energy loss and carrier diffusion in a semiconductor nanowire array using 4D electron microscopy," Adv. Mater. 28(25), 5106-5111 (2016).

142. J. I. Khan et al., "Enhanced optoelectronic performance of a passivated nanowire-based device: key information from real-space imaging using 4D electron microscopy," Small 12(17), 2313-2320 (2016). 
143. A. Armstrong et al., "GaN nanowire surface state observed using deep level optical spectroscopy," Appl. Phys. Lett. 96(16), 163106 (2010).

144. R. Calarco et al., "Surface-induced effects in GaN nanowires," J. Mater. Res. 26(17), 2157-2168 (2011).

145. P. Varadhan et al., "Surface passivation of GaN nanowires for enhanced photoelectrochemical water-splitting," Nano Lett. 17(3), 1520-1528 (2017).

146. M. Ebaid et al., "Unbiased photocatalytic hydrogen generation from pure water on stable Ir-treated $\mathrm{In}_{0.33} \mathrm{Ga}_{0.67} \mathrm{~N}$ nanorods," Nano Energy 37, 158-167 (2017).

147. H. P. T. Nguyen et al., "Nonradiative recombination mechanism in phosphor-free GaNbased nanowire white light emitting diodes and the effect of ammonium sulfide surface passivation," ECS Trans. 53(2), 93-100 (2013).

148. N. Tajik et al., "Improved conductivity and long-term stability of sulfur-passivated n-GaAs nanowires," Appl. Phys. Lett. 100(20), 203122 (2012).

149. M. H. Sun et al., "Removal of surface states and recovery of band-edge emission in InAs nanowires through surface passivation," Nano Lett. 12, 3378-3384 (2012).

150. N. Tajik et al., "Photoluminescence model of sulfur passivated p-InP nanowires," Nanotechnology 23(31), 315703 (2012).

151. D. Rudolph et al., "Spontaneous alloy composition ordering in GaAs-AlGaAs core-shell nanowires," Nano Lett. 13(4), 1522-1527 (2013).

152. A. C. E. Chia et al., "Electrical transport and optical model of GaAs-AlInP core-shell nanowires," J. Appl. Phys. 111(9), 094319 (2012).

153. Q. Wang et al., "High efficiency ultraviolet emission from $\mathrm{Al}_{x} \mathrm{Ga}_{1-x} \mathrm{~N}$ core-shell nanowire heterostructures grown on Si (111) by molecular beam epitaxy," Appl. Phys. Lett. 101(4), 043115 (2012).

154. H. P. T. Nguyen et al., "Breaking the carrier injection bottleneck of phosphor-free nanowire white light-emitting diodes," Nano Lett. 13(11), 5437-5442 (2013).

155. H. P. T. Nguyen et al., "Engineering the carrier dynamics of InGaN nanowire white light-emitting diodes by distributed p-AlGaN electron blocking layers," Sci. Rep. 5, 1-8 (2015).

156. N. Jiang et al., "Enhanced minority carrier lifetimes in GaAs/AlGaAs core-shell nanowires through shell growth optimization," Nano Lett. 13(11), 5135-5140 (2013).

157. O. Brandt et al., "Statistical analysis of the shape of one-dimensional nanostructures: determining the coalescence degree of spontaneously formed GaN nanowires," Cryst. Growth Des. 14(5), 2246-2253 (2014).

158. C. Pfüller et al., "Statistical analysis of excitonic transitions in single, free-standing GaN nanowires: probing impurity incorporation in the Poissonian limit," Nano Res. 3(12), 881-888 (2010).

159. F. Limbach et al., "Current path in light emitting diodes based on nanowire ensembles," Nanotechnology 23(46), 465301 (2012).

160. S. Jahangir et al., "Red-emitting $(\lambda=610 \mathrm{~nm}) \mathrm{In}_{0.51} \mathrm{Ga}_{0.49} \mathrm{~N} / \mathrm{GaN}$ disk-in-nanowire light emitting diodes on silicon," IEEE J. Quantum Electron. 50(7), 530-537 (2014).

161. D. Mikulik et al., "Conductive-probe atomic force microscopy as a characterization tool for nanowire-based solar cells," Nano Energy 41, 566-572 (2017).

162. J.-S. Hwang et al., "Photocurrent mapping in high-efficiency radial $\mathrm{p}-\mathrm{n}$ junction silicon nanowire solar cells using atomic force microscopy," J. Phys. Chem. C 115(44), 2198121986 (2011).

163. N. J. Ku et al., "Energy harvesting from the obliquely aligned InN nanowire array with a surface electron-accumulation layer," Adv. Mater. 25(6), 861-866 (2013).

164. C. H. Wang et al., "Effects of free carriers on piezoelectric nanogenerators and piezotronic devices made of GaN nanowire arrays," Small 10(22), 4718-4725 (2014).

165. G. Liu et al., "Nanogenerators based on vertically aligned InN nanowires," Nanoscale 8(4), 2097-2106 (2016).

166. N. J. $\mathrm{Ku}$ et al., "Crystal face-dependent nanopiezotronics of an obliquely aligned $\mathrm{InN}$ nanorod Array," Nano Lett. 12(2), 562-568 (2012).

167. B. J. May et al., "Nanoscale current uniformity and injection efficiency of nanowire light emitting diodes," Appl. Phys. Lett. 112(9), 093107 (2018). 
168. B. J. May et al., "Nanoscale electronic conditioning for improvement of nanowire LED efficiency," ACS Nano 12(4), 3551-3556 (2018).

169. T. Yanagisawa et al., "Degradation of InGaN blue light-emitting diodes under continuous and low-speed pulse operations," Microelectron. Reliab. 43(6), 977-980 (2003).

170. T. Yanagisawa, "Estimation of the degradation of InGaN/AlGaN blue light-emitting diodes," Microelectron. Reliab. 37(8), 1239-1241 (1997).

171. Z. Z. Chen et al., "Study on the stability of the high-brightness white LED," Phys. Status Solidi Basic Res. 241(12), 2664-2667 (2004).

172. A. Uddin et al., "Study of degradation mechanism of blue light emitting diodes," Thin Solid Films 483(1-2), 378-381 (2005).

173. Z. L. Li et al., "Reliability study on green InGaN/GaN light emitting diodes," J. Phys. Conf. Ser. 209, 012065 (2010).

174. M. Meneghini et al., "A review on the reliability of GaN-based LEDs," IEEE Trans. Device Mater. Reliab. 8(2), 323-331 (2008).

175. F. Rossi et al., "Influence of short-term low current dc aging on the electrical and optical properties of InGaN blue light-emitting diodes," J. Appl. Phys. 99(5), 053104 (2006).

176. X. A. Cao et al., "Defect generation in $\mathrm{InGaN} / \mathrm{GaN}$ light-emitting diodes under forward and reverse electrical stresses," Microelectron. Reliab. 43(12), 1987-1991 (2003).

177. N. Renso et al., "Understanding the degradation processes of GaN based LEDs submitted to extremely high current density," Microelectron. Reliab. 76-77, 556-560 (2017).

178. M. Meneghini et al., "A review on the physical mechanisms that limit the reliability of GaN-based LEDs," IEEE Trans. Electron Devices 57(1), 108-118 (2010).

179. N. Trivellin et al., "A review on the reliability of GaN-based laser diodes," in IEEE Int. Reliability Physics Symp. Proc., pp. 1-6 (2010).

180. M. H. Chang et al., "Light emitting diodes reliability review," Microelectron. Reliab. 52(5), 762-782 (2012).

181. C. G. Moe et al., "Current-induced degradation of high performance deep ultraviolet light emitting diodes," Appl. Phys. Lett. 96(21), 213512 (2010).

182. M. Meneghini et al., "Analysis of the physical processes responsible for the degradation of deep-ultraviolet light emitting diodes," Appl. Phys. Lett. 97(14), 143506 (2010).

183. M. Meneghini et al., "Reliability of deep-UV light-emitting diodes," IEEE Trans. Device Mater. Reliab. 8(2), 248-254 (2008).

184. D. Monti et al., "Defect-generation and diffusion in (In) AlGaN-based UV-B LEDs," Proc. SPIE 10554, 1055410 (2018).

185. J. Glaab et al., "Degradation effects of the active region in UV-C light-emitting diodes," J. Appl. Phys. 123(10), 104502 (2018).

186. D. Monti et al., "Defect-related degradation of AlGaN-based UV-B LEDs," IEEE Trans. Electron. Devices 64(1), 200-205 (2017).

187. Y. Xi et al., "Junction and carrier temperature measurements in deep-ultraviolet lightemitting diodes using three different methods," Appl. Phys. Lett. 86(3), 031907 (2005).

188. Y. Xi et al., "Junction-temperature measurement in GaN ultraviolet light-emitting diodes using diode forward voltage method," Appl. Phys. Lett. 85(12), 2163-2165 (2004).

189. M. Tangi et al., "Type-I band alignment at $\mathrm{MoS}_{2} / \mathrm{In}_{0.15} \mathrm{Al}_{0.85} \mathrm{~N}$ lattice matched heterojunction and realization of $\mathrm{MoS}_{2}$ quantum well," Appl. Phys. Lett. 111(9), 092104 (2017).

190. M. A. Khan et al., "High-responsivity photoconductive ultraviolet sensors based on insulating single-crystal GaN epilayers," Appl. Phys. Lett. 60(23), 2917-2919 (1992).

191. E. Monroy et al., "Wide-bandgap semiconductor ultraviolet photodetectors," Semicond. Sci. Technol. 18(4), R33 (2003).

192. R. McClintock et al., "High quantum efficiency AlGaN solar-blind p-i-n photodiodes," Appl. Phys. Lett. 84(8), 1248-1250 (2004).

193. T. Tut et al., "Solar-blind AlGaN-based p-i-n photodetectors with high breakdown voltage and detectivity," Appl. Phys. Lett. 92(10), 103502 (2008).

194. T. Deguchi et al., "Quantum-confined stark effect in an AlGaN/GaN/AlGaN single quantum well structure," Jpn. J. Appl. Phys. 38(8B), L914-L916 (1999).

195. K. M. Azizur-Rahman et al., "Wavelength-selective absorptance in GaAs, InP and InAs nanowire arrays," Nanotechnology 26(29), 295202 (2015). 
196. R. R. Lapierre et al., "A review of III-V nanowire infrared photodetectors and sensors," J. Phys. D. Appl. Phys. 50(12), 123001 (2017).

197. L. Rigutti et al., "GaN nanowire-based ultraviolet photodetectors," in Wide Band Gap Semiconductor Nanowires 2: Heterostructures and Optoelectronic Devices, V. Consonni and G. Feuillet, Eds., pp. 179-202, ISTE Ltd and John Wiley \& Sons, Inc., Great Britain (2014).

198. Z. Fan et al., "Electrical and photoconductive properties of vertical $\mathrm{ZnO}$ nanowires in high density arrays," Appl. Phys. Lett. 89(21), 213110 (2006).

199. D. Park et al., "Photoconductivity of vertically aligned $\mathrm{ZnO}$ nanoneedle array," J. Vac. Sci. Technol., B 26(6), 1933-1936 (2008).

200. J. S. Lee et al., "Photoresponses of $\mathrm{ZnO}$ nanobridge devices fabricated using a single-step thermal evaporation method," Sens. Actuators, B 126(1), 73-77 (2007).

201. C. L. Hsu et al., "Ultraviolet photodetectors with low temperature synthesized vertical $\mathrm{ZnO}$ nanowires," Chem. Phys. Lett. 416(1-3), 75-78 (2005).

202. C. H. Chen et al., "High detectivity mechanism of ZnO-based nanorod ultraviolet photodetectors," IEEE Photonics Technol. Lett. 25(4), 348-351 (2013).

203. C. Soci et al., "ZnO nanowire UV photodetectors with high internal gain," Nano Lett. 7(4), 1003-1009 (2007).

204. F. González-Posada et al., "Responsivity and photocurrent dynamics in single GaN nanowires," Phys. Status Solidi Curr. Top. Solid State Phys. 9(3-4), 642-645 (2012).

205. M. Den Hertog et al., "Single GaN-based nanowires for photodetection and sensing applications," Jpn. J. Appl. Phys. 52(11 Part 2), 1-6 (2013).

206. X. Wang et al., "Ultrafast, superhigh gain visible-blind UV detector and optical logic gates based on nonpolar a-axial GaN nanowire," Nanoscale 6(20), 12009-12017 (2014).

207. A. V. Babichev et al., "GaN nanowire ultraviolet photodetector with a graphene transparent contact," Appl. Phys. Lett. 103(20), 201103 (2013).

208. S. Kang et al., "Ultraviolet-C photodetector fabricated using Si-doped n-AlGaN nanorods grown by MOCVD," ACS Photonics 4(10), 2595-2603 (2017).

209. S. Kang et al., "Synthesis of n-AlGaN nanoflowers by MOCVD for high-performance ultraviolet-C photodetectors," J. Mater. Chem. C 6(5), 1176-1186 (2018).

210. B. Yuan et al., "High photosensitivity and low dark current of photoconductive semiconductor switch based on ZnO single nanobelt," Solid. State. Electron. 55(1), 49-53 (2011).

211. N. Biyikli et al., "Solar-blind AlGaN-based p-i-n photodiodes with low dark current and high detectivity," IEEE Photonics Technol. Lett. 16(7), 1718-1720 (2004).

Jung-Wook Min received his PhD in applied physics from GIST, Republic of Korea, in 2016. He joined Ooi-group at KAUST as a postdoctoral research fellow in 2017. His research mainly focuses on the PA-MBE growth on III-nitride nanowire arrays for UV light-emitting diodes and solar water-splitting.

Davide Priante received his BS degree in material science and engineering from Padua University, Italy, in 2013, and his master's degree in electrical engineering from KAUST in 2015. He is currently pursuing his PhD in Photonics Lab under the supervision of Prof. Ooi with a research focus on III-nitride wide-bandgap optoelectronic semiconductor devices. $\mathrm{He}$ is a member of OSA and SPIE.

Malleswararao Tangi received his $\mathrm{PhD}$ in physics from Jawaharlal Nehru Center for Advanced Scientific Research, India, in 2015. He joined Ooi-group at KAUST as a postdoctoral research fellow in 2015. Currently, he is working on molecular beam epitaxy growth of III-N material and optoelectronic devices.

Guangyu Liu received her $\mathrm{PhD}$ in electrical engineering from Lehigh University, Pennsylvania, USA, in 2013 with the research focus on III-nitride semiconductors for solid-state lighting. Prior to KAUST, she worked as a process development engineer at Intel Corp., USA, from 2013 to 2016. She is currently a research scientist with Ooi-group at KAUST. Her current research area is III-nitride semiconductor-based integrated devices and systems for communication and sensing applications. 
Chun Hong Kang received his BEng and MSc degrees in electrical and electronics engineering from the Universiti Teknologi PETRONAS, Malaysia, in 2014 and 2016, respectively. He joined Photonics Laboratory at KAUST under the supervision of Prof. Ooi in 2017.

Aditya Prabaswara received his bachelor's degree in telecommunication engineering from the Institut Teknologi Bandung, Indonesia, and his MS degree in electrical engineering from KAIST, Republic of Korea. He joined KAUST as a PhD candidate in 2014 under the supervision of Prof. Ooi. Currently, he is working on III-nitride nanowire-based optoelectronics.

Chao Zhao received his $\mathrm{PhD}$ in microelectronics and solid-state electronics from the Chinese Academy of Sciences in 2009. His PhD research focused on the MBE growth and characterization of InAs/GaAs and $\mathrm{GaAs} / \mathrm{AlGaAs}$ nanostructures. He is currently working on molecular beam epitaxy (MBE) growth of III-N material and optoelectronic device at KAUST. He has authored, coauthored, and delivered more than 80 journal articles, invited talks, and conference articles. He is a senior member of IEEE and serves as a reviewer for various international journals, such as Optics Express, Scientific Reports, Langmuir, and Nanotechnology. He has also been the session chair for several international conferences.

Latifah Al-Maghrabi graduated with her BS degree in electrical engineering from Georgia Tech, Atlanta, Georgia, USA, in May 2018. In the Fall of 2018, she is looking forward to pursuing her master's degree in electrical engineering at KAUST. In 2016, she was a summer intern at Aramco Services Company, Houston, Texas, USA, and she was a summer intern at KAUST in 2017. Her current interests include device physics, optoelectronics, and PCB design.

Yazeed Alaskar received his $\mathrm{PhD}$ in electrical engineering from the University of CaliforniaLos Angeles (UCLA). He is currently working as an assistant professor at the National Nanotechnology Research Center of KACST. He is also working as a principal investigator at the Center of Excellence for Green Nanotechnologies (CEGN) at UCLA. His research interests include epitaxial growth of III/V semiconductors, optoelectronic devices and lasers. In addition, he is interested in commercializing innovative technologies and transitioning them from the lab to market.

Abdulrahman M. Albadri received his $\mathrm{PhD}$ from Vanderbilt University, Nashville, Tennessee, USA. He is the manager of the National Center for Nanotechnology and Advanced Materials, KACST, Saudi Arabia. His current research activities include solar cells (GaAs solar cells and perovskite solar cells), GaN LED/laser devices, and basic research on oxide/nitride semiconductors.

\begin{abstract}
Ahmed Y. Alyamani received his $\mathrm{PhD}$ in physics from the University of Sheffield, UK. He is currently the director of the Centre of Excellence for Solid State Lighting (CSSL) at KACST and a member of the Strategic Plan Committee of Nanotechnology and Advanced Materials Programs in Saudi Arabia. His current interests include growth, fabrication, and characterization of wide-bandgap semiconductors for LED, ferromagnetic, and photovoltaic applications.
\end{abstract}

Tien Khee Ng received his MEng degree and $\mathrm{PhD}$ in electrical and electronic engineering from Nanyang Technological University, Singapore, in 2001 and 2005, respectively. Currently, he is a senior research scientist in Ooi-Group at KAUST, Saudi Arabia, and the coprincipal-investigator responsible for molecular beam epitaxy thrust for the KACST Technology Innovation Center for Solid-State Lighting at KAUST. He is a senior member of IEEE, member of SPIE, and senior member of OSA.

Boon S. Ooi received his $\mathrm{PhD}$ in electronics and electrical engineering from the University of Glasgow. He is a professor of electrical engineering at KAUST. His research is concerned with the study of semiconductor lasers, LEDs, and photonic integrated circuits for applications in energy efficient lighting and visible light communications. He is an associate editor of Optics Express and Journal of Nanophotonics. He is a fellow of OSA, SPIE, and IoP (UK). 